\title{
SUBGRADIENT ESTIMATE AND LIOUVILLE-TYPE THEOREM FOR THE CR HEAT EQUATION ON HEISENBERG GROUPS*
}

\author{
SHU-CHENG CHANG ${ }^{\dagger}$, JINGZHI TIE ${ }^{\ddagger}$, AND CHIN-TUNG WU§
}

\begin{abstract}
In this paper, we first get a subgradient estimate of the $\mathrm{CR}$ heat equation on a closed pseudohermitian $(2 n+1)$-manifold. Secondly, by deriving the CR version of sub-Laplacian comparison theorem on an $(2 n+1)$-dimensional Heisenberg group $H^{n}$, we are able to establish a subgradient estimate and then the Liouville-type theorem for the CR heat equation on $H^{n}$.
\end{abstract}

Key words. Subgradient estimate, Liouville-type Theorem, Heat Kernel, Pseudohermitian manifold, Heisenberg Group, CR-pluriharmonic, CR-Paneitz operator, Sub-Laplacian, Li-Yau Harnack inequality.

AMS subject classifications. Primary 32V05, 32V20; Secondary 53C56

1. Introduction. In the paper of $[Y]$, S.-T. Yau derived a gradient estimate for positive harmonic functions on a complete noncompact Riemannian manifold. As a consequence, Liouville-type theorems can be proved for manifolds of nonnegative Ricci curvature. Moreover, in the paper of [LY], P. Li and S.-T. Yau established the parabolic Li-Yau gradient estimate and Li-Yau Harnack inequality for the positive solution of the heat equation on a complete Riemannian manifold.

However for a pseudohermitian $(2 n+1)$-manifold $(M, J, \theta)$, the corresponding estimates are not clear due to a lack of sub-Laplacian comparison theorem and CR Bochner formula. In this paper, we consider the CR heat equation (1.6) with respect to the sub-Laplacian on $(M, J, \theta)$. By using the arguments of [LY] and CR Bochner formula (2.1), we are able to derive the CR version of parabolic Li-Yau gradient estimate and the so-called reversed Li-Yau Harnack inequality for the positive solution of $\mathrm{CR}$ heat equation. Then by combining the standard parabolic Li-Yau gradient estimate, we derive a subgradient estimate of the CR heat equation on closed pseudohermitian $(2 n+1)$-manifolds. Moreover, by deriving the $\mathrm{CR}$ version of sub-Laplacian comparison theorem on $(2 n+1)$-dimensional Heisenberg groups $H^{n}$, we are able to establish the subgradient estimate and the Liouville-type theorem for the $\mathrm{CR}$ heat equation on $H^{n}$.

The main key step is to derive the CR version of Bochner formula. This formula (2.1) involving a third order operator $P$ which characterizes CR-pluriharmonic functions ([L1]), is hard to control. However after integrating by parts (see 1.5), we are able to relate this extra term to the CR Paneitz operator $P_{0}$.

We first give a brief introduction to pseudohermitian geometry (see [L1] for more details). Let $(M, \xi)$ be a $(2 n+1)$-dimensional, orientable, contact manifold with contact structure $\xi, \operatorname{dim}_{R} \xi=2 n$. A CR structure compatible with $\xi$ is an endomorphism $J: \xi \rightarrow \xi$ such that $J^{2}=-1$. We also assume that $J$ satisfies the following integrability condition: If $X$ and $Y$ are in $\xi$, then so is $[J X, Y]+[X, J Y]$

\footnotetext{
${ }^{*}$ Received January 18, 2009; accepted for publication December 22, 2009. Research supported in part by NSC of Taiwan.

${ }^{\dagger}$ Department of Mathematics, National Taiwan University, Taipei 10617, Taiwan, R.O.C. (scchang @math.ntu.edu.tw).

${ }^{\ddagger}$ Department of Mathematics, University of Georgia, Athens, GA 30602-7403, U.S.A. (jtie@ math.uga.edu).

$\S$ Department of Applied Mathematics, National Pingtung University of Education, Pingtung, Taiwan 90003, R.O.C. (ctwu@mail.npue.edu.tw).
} 
and $J([J X, Y]+[X, J Y])=[J X, J Y]-[X, Y]$. A CR structure $J$ can extend to $\mathbb{C} \otimes \xi$ and decomposes $\mathbb{C} \otimes \xi$ into the direct sum of $T_{1,0}$ and $T_{0,1}$ which are eigenspaces of $J$ with respect to $i$ and $-i$, respectively. A manifold $M$ with a CR structure is called a CR manifold. A pseudohermitian structure compatible with $\xi$ is a $C R$ structure $J$ compatible with $\xi$ together with a choice of contact form $\theta$. Such a choice determines a unique real vector field $T$ transverse to $\xi$, which is called the the characteristic vector field of $\theta$, such that $\theta(T)=1$ and $\mathcal{L}_{T} \theta=0$ or $d \theta(T, \cdot)=0$. Let $\left\{T, Z_{\alpha}, Z_{\bar{\alpha}}\right\}$ be a frame of $T M \otimes \mathbb{C}$, where $Z_{\alpha}$ is any local frame of $T_{1,0}, Z_{\bar{\alpha}}=\overline{Z_{\alpha}} \in T_{0,1}$ and $T$ is the characteristic vector field. Then $\left\{\theta, \theta^{\alpha}, \theta^{\bar{\alpha}}\right\}$, which is the coframe dual to $\left\{T, Z_{\alpha}, Z_{\bar{\alpha}}\right\}$, satisfies

$$
d \theta=i h_{\alpha \bar{\beta}} \theta^{\alpha} \wedge \theta^{\bar{\beta}}
$$

for some hermitian matrix of functions $\left(h_{\alpha \bar{\beta}}\right)$. Actually we can always choose $Z_{\alpha}$ such that $h_{\alpha \bar{\beta}}=\delta_{\alpha \beta}$; hence, throughout this paper, we assume $h_{\alpha \bar{\beta}}=\delta_{\alpha \beta}$.

The Levi form $\langle,\rangle_{L_{\theta}}$ is the Hermitian form on $T_{1,0}$ defined by

$$
\langle Z, W\rangle_{L_{\theta}}=-i\langle d \theta, Z \wedge \bar{W}\rangle .
$$

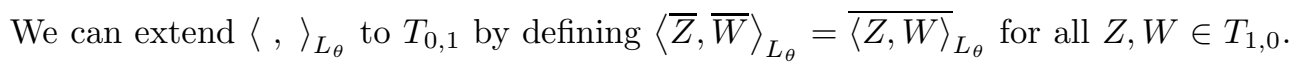
The Levi form induces naturally a Hermitian form on the dual bundle of $T_{1,0}$, denoted by $\langle,\rangle_{L_{\theta}^{*}}$, and hence on all the induced tensor bundles. Integrating the Hermitian form (when acting on sections) over $M$ with respect to the volume form $d \mu=\theta \wedge d \theta$, we get an inner product on the space of sections of each tensor bundle. We denote the inner product by the notation $\langle$,$\rangle . For example$

$$
\langle\varphi, \psi\rangle=\int_{M} \varphi \bar{\psi} d \mu
$$

for functions $\varphi$ and $\psi$.

The pseudohermitian connection of $(J, \theta)$ is the connection $\nabla$ on $T M \otimes \mathbb{C}$ (and extended to tensors) given in terms of a local frame $Z_{\alpha} \in T_{1,0}$ by

$$
\nabla Z_{\alpha}=\theta_{\alpha}{ }^{\beta} \otimes Z_{\beta}, \quad \nabla Z_{\bar{\alpha}}=\theta_{\bar{\alpha}}^{\bar{\beta}} \otimes Z_{\bar{\beta}}, \quad \nabla T=0,
$$

where $\theta_{\alpha}{ }^{\beta}$ are the 1 -forms uniquely determined by the following equations:

$$
\begin{aligned}
d \theta^{\beta} & =\theta^{\alpha} \wedge \theta_{\alpha}{ }^{\beta}+\theta \wedge \tau^{\beta}, \\
0 & =\tau_{\alpha} \wedge \theta^{\alpha}, \\
0 & =\theta_{\alpha}{ }^{\beta}+\theta_{\bar{\beta}^{\bar{\alpha}}}{ }^{\bar{\alpha}},
\end{aligned}
$$

We can write (by Cartan lemma) $\tau_{\alpha}=A_{\alpha \gamma} \theta^{\gamma}$ with $A_{\alpha \gamma}=A_{\gamma \alpha}$. The curvature of the Webster-Stanton connection, expressed in terms of the coframe $\left\{\theta=\theta^{0}, \theta^{\alpha}, \theta^{\bar{\alpha}}\right\}$, is

$$
\begin{aligned}
\Pi_{\beta}{ }^{\alpha} & =\bar{\Pi}_{\bar{\beta}}^{\bar{\alpha}}=d \theta_{\beta}{ }^{\alpha}-\theta_{\beta}{ }^{\gamma} \wedge \theta_{\gamma}{ }^{\alpha}, \\
\Pi_{0}{ }^{\alpha} & =\Pi_{\alpha}{ }^{0}=\Pi_{0}{ }^{\bar{\beta}}=\Pi_{\bar{\beta}}{ }^{0}=\Pi_{0}{ }^{0}=0 .
\end{aligned}
$$

Webster showed that $\Pi_{\beta}{ }^{\alpha}$ can be written

$$
\Pi_{\beta}{ }^{\alpha}=R_{\beta}{ }^{\alpha}{ }_{\rho \bar{\rho}} \theta^{\rho} \wedge \theta^{\bar{\sigma}}+W_{\beta}{ }_{\rho}^{\alpha} \theta^{\rho} \wedge \theta-W^{\alpha}{ }_{\beta \bar{\rho}} \theta^{\bar{\rho}} \wedge \theta+i \theta_{\beta} \wedge \tau^{\alpha}-i \tau_{\beta} \wedge \theta^{\alpha}
$$


where the coefficients satisfy

$$
R_{\beta \bar{\alpha} \rho \bar{\sigma}}=\overline{R_{\alpha \bar{\beta} \sigma \bar{\rho}}}=R_{\bar{\alpha} \beta \bar{\sigma} \rho}=R_{\rho \bar{\alpha} \beta \bar{\sigma}}, \quad W_{\beta \bar{\alpha} \gamma}=W_{\gamma \bar{\alpha} \beta} .
$$

We will denote components of covariant derivatives with indices preceded by comma; thus write $A_{\alpha \beta, \gamma}$. The indices $\{0, \alpha, \bar{\alpha}\}$ indicate derivatives with respect to $\left\{T, Z_{\alpha}, Z_{\bar{\alpha}}\right\}$. For derivatives of a scalar function, we will often omit the comma, for instance, $f_{\alpha}=Z_{\alpha} f, f_{\alpha \bar{\beta}}=Z_{\bar{\beta}} Z_{\alpha} f-\theta_{\alpha}^{\gamma}\left(Z_{\bar{\beta}}\right) Z_{\gamma} f, f_{0}=T f$ for a (smooth) function.

For a real function $f$, the subgradient $\nabla_{b}$ is defined by $\nabla_{b} f \in \xi$ and $\left\langle Z, \nabla_{b} f\right\rangle_{L_{\theta}}=$ $d f(Z)$ for all vector fields $Z$ tangent to contact plane. Locally $\nabla_{b} f=\sum_{\alpha} f_{\bar{\alpha}} Z_{\alpha}+f_{\alpha} Z_{\bar{\alpha}}$. We can use the connection to define the subhessian as the complex linear map

$$
\left(\nabla^{H}\right)^{2} f: T_{1,0} \oplus T_{0,1} \rightarrow T_{1,0} \oplus T_{0,1},
$$

by

$$
\left(\nabla^{H}\right)^{2} f(Z)=\nabla_{Z} \nabla_{b} f
$$

Also

$$
\Delta_{b} f=\operatorname{Tr}\left(\left(\nabla^{H}\right)^{2} f\right)=\sum_{\alpha}\left(f_{\alpha \bar{\alpha}}+f_{\bar{\alpha} \alpha}\right) .
$$

The Webster-Ricci tensor and the torsion tensor on $T_{1,0}$ are defined by

$$
\operatorname{Ric}(X, Y)=R_{\alpha \bar{\beta}} X^{\alpha} Y^{\bar{\beta}},
$$

and

$$
\operatorname{Tor}(X, Y)=i \sum_{\alpha, \beta}\left(A_{\bar{\alpha} \bar{\beta}} X^{\bar{\alpha}} Y^{\bar{\beta}}-A_{\alpha \beta} X^{\alpha} Y^{\beta}\right),
$$

where $X=X^{\alpha} Z_{\alpha}, Y=Y^{\beta} Z_{\beta}, R_{\alpha \bar{\beta}}=R_{\gamma}{ }_{\alpha \bar{\beta}}$. The Webster scalar curvature is $R=R_{\alpha}{ }^{\alpha}=h^{\alpha \bar{\beta}} R_{\alpha \bar{\beta}}$.

Next we recall some definitions.

Definition 1.1. (i) A piecewise smooth curve $\gamma:[0,1] \rightarrow M$ is said to be horizontal if $\gamma^{\prime}(t) \in \xi$ whenever $\gamma^{\prime}(t)$ exists. The length of $\gamma$ is then defined by

$$
l(\gamma)=\int_{0}^{1}\left\langle\gamma^{\prime}(t), \gamma^{\prime}(t)\right\rangle^{\frac{1}{2}} d t .
$$

The Carnot-Carathéodory distance between two points $p, q \in M$ is

$$
d_{c}(p, q)=\inf \left\{l(\gamma) \mid \gamma \in C_{p, q}\right\},
$$

where $C_{p, q}$ is the set of all horizontal curves joining $p$ and $q$.

(ii) By Chow connectivity theorem [Cho], there always exists a horizontal curve joining $p$ and $q$, so the distance is finite. We say $M$ is complete if it is complete as a metric space.

Definition 1.2. A smooth real-valued function $u$ in $M$ is said to be CRpluriharmonic function if for any point $p \in M$, there is an open neighborhood $U$ of $p$ in $M$ and a smooth real-valued function $v$ on $U$ such that $\bar{\partial}_{b}(u+i v)=0$. 
Definition 1.3. ([L1]) Let $\left(M^{2 n+1}, J, \theta\right)$ be a complete pseudohermitian manifold. Define

$$
P \varphi=\sum_{\alpha=1}^{n}\left(\varphi \bar{\alpha}^{\bar{\alpha}}{ }_{\beta}+i n A_{\beta \alpha} \varphi^{\alpha}\right) \theta^{\beta}=\left(P_{\beta} \varphi\right) \theta^{\beta}, \quad \beta=1,2, \cdots, n
$$

which is an operator that characterizes CR-pluriharmonic functions. Here

$$
P_{\beta} \varphi=\sum_{\alpha=1}^{n}\left(\varphi \bar{\alpha}^{\bar{\alpha}}{ }+i n A_{\beta \alpha} \varphi^{\alpha}\right)
$$

and $\bar{P} \varphi=\left(\bar{P}_{\beta} \varphi\right) \theta^{\bar{\beta}}$, the conjugate of $P$. Moreover we define

$$
P_{0} \varphi=4\left(\delta_{b}(P \varphi)+\bar{\delta}_{b}(\bar{P} \varphi)\right)
$$

which is the so-called CR Paneitz operator $P_{0}$. Here $\delta_{b}$ is the divergence operator that takes $(1,0)$-forms to functions by $\delta_{b}\left(\sigma_{\alpha} \theta^{\alpha}\right)=\sigma_{\alpha}{ }^{\alpha}$ and $\bar{\delta}_{b}\left(\sigma_{\bar{\alpha}} \theta^{\bar{\alpha}}\right)=\sigma_{\bar{\alpha}},{ }^{\bar{\alpha}}$. If we define $\partial_{b} \varphi=\varphi_{\alpha} \theta^{\alpha}$ and $\bar{\partial}_{b} \varphi=\varphi_{\bar{\alpha}} \theta^{\bar{\alpha}}$, then the formal adjoint of $\partial_{b}$ on functions (with respect to the Levi form and the volume form $d \mu$ ) is $\partial_{b}^{*}=-\delta_{b}$.

We observe that if $(M, J, \theta)$ is a closed pseudohermitian $(2 n+1)$-manifold, then

$$
-\int_{M}\left\langle P \varphi+\bar{P} \varphi, d_{b} \varphi\right\rangle d \mu=\frac{1}{4} \int_{M} P_{0} \varphi \cdot \varphi d \mu .
$$

In particular if $(M, J, \theta)$ has zero torsion, we have

$$
P_{0} \varphi=\mathcal{L}_{n} \mathcal{L}_{\bar{n}}=\left[\Delta_{b}^{2} \varphi+n^{2} T^{2} \varphi\right] .
$$

Here

$$
\mathcal{L}_{n} \varphi=-\Delta_{b} \varphi+i n T \varphi=-2 \varphi \bar{\alpha}^{\bar{\alpha}} .
$$

For the details about these operators, the reader can make reference to [GL], $[\mathrm{H}]$ and [L1].

REMARK 1.1. ([H], $[G L])$ (i) Let $(M, J, \theta)$ be a closed pseudohermitian $(2 n+1)$ manifold with $n \geq 2$. Then a smooth real-valued function $f$ satisfies $P_{0} f=0$ on $M$ if and only if $P_{\beta} f=0$ on $M$. It holds also for a closed pseudohermitian 3-manifold of zero torsion.

(ii) Let $P_{\beta} f=0$. If $M$ is the boundary of a connected strictly pseudoconvex domain $\Omega \subset C^{n+1}$, then $f$ is the boundary value of a pluriharmonic function $u$ in $\Omega$. That is, $\partial \bar{\partial} u=0$ in $\Omega$. Moreover, if $\Omega$ is simply connected, there exists a holomorphic function $w$ in $\Omega$ such that $\operatorname{Re}(w)=u$ and $\left.u\right|_{M}=f$.

In this paper, we consider the positive solution $u(x, t)$ of the $\mathrm{CR}$ heat equation with respect to the sub-Laplacian

$$
\frac{\partial}{\partial t} u(x, t)=\Delta_{b} u(x, t)
$$

on $M \times[0, T)$. 
Proposition 1.1. Let $(M, J, \theta)$ be a closed pseudohermitian $(2 n+1)$-manifold. If $u(x, t)$ is the positive smooth solution of (1.6) on $M \times[0, \infty)$. Suppose that

$$
[2 \operatorname{Ric}-(n+2) \operatorname{Tor}](Z, Z) \geq-l_{0}|Z|^{2},
$$

for all $Z \in T_{1,0}$ and $l_{0}$ is a nonnegative constant. Then the function

$$
G=t\left[\left|\nabla_{b} \varphi\right|^{2}+\left(1+\frac{2}{n}\right) \varphi_{t}\right]
$$

satisfies the inequality

$$
\begin{aligned}
\left(\Delta_{b}-\frac{\partial}{\partial t}\right) G \geq & -\frac{2 n}{n+2}\left\langle\nabla_{b} \varphi, \nabla_{b} G\right\rangle \\
& +\frac{2 n}{(n+1)(n+2)^{2} t} G\left(G-\frac{(n+1)(n+2)^{2}}{2 n}\right) \\
& -l_{0} t\left|\nabla_{b} \varphi\right|^{2}-\frac{8}{n} t u^{-2}\left\langle P u+\bar{P} u, d_{b} u\right\rangle_{L_{\theta}^{*}} .
\end{aligned}
$$

Let $u(x, t)$ be a positive solution of (1.6) on $M \times[0, \infty)$. In section 2 , it is proved that if $P_{\beta} u=0$ at $t=0$, then $P_{\beta} u=0$ for all $t$ on a closed pseudohermitian $(2 n+1)$ manifold of zero torsion. Then the extra term of CR Bochner formula (2.1) becomes

$$
\left\langle P u+\bar{P} u, d_{b} u\right\rangle=0
$$

on $M \times[0, \infty)$.

Now by using the arguments of [LY], (2.1) and (1.8), we are able to derive the CR version of parabolic Li-Yau gradient estimate for the positive solution $u(x, t)$ of (1.6) on $M \times[0, \infty)$.

Corollary 1.2. Let $(M, J, \theta)$ be a closed pseudohermitian $(2 n+1)$-manifold of zero torsion and nonnegative pseudohermitian Ricci tensors. If $u(x, t)$ is the positive solution of (1.6) on $M \times[0, \infty)$ such that

$$
P_{\beta} u=0
$$

at $t=0$. Then $u$ satisfies the estimate

$$
\frac{\left|\nabla_{b} u\right|^{2}}{u^{2}}+\frac{n+2}{n} \frac{u_{t}}{u} \leq \frac{(n+1)(n+2)^{2}}{2 n} \frac{1}{t}
$$

on $M \times[0, \infty)$.

By combining the result of [CY] and Corollary 1.2, we get the following subgradient estimate of the logarithm of a positive solution to (1.6).

TheOrem 1.3. Let $(M, J, \theta)$ be a closed pseudohermitian $(2 n+1)$-manifold of zero torsion and nonnegative pseudohermitian Ricci tensor. If $u(x, t)$ is the positive solution of (1.6) on $M \times[0, \infty)$ such that

$$
P_{\beta} u=0, \quad \beta=1,2, \cdots, n
$$

at $t=0$. Then there exist constants $C_{1}, C_{2}$ such that $u$ satisfies the subgradient estimate

$$
t\left|\nabla_{b} \log u\right|^{2} \leq C_{1}+C_{2} t
$$


on $M \times[0, \infty)$.

Note that the arguments of $[\mathrm{LY}]$ can be extended easily to complete noncompact pseudohermitian $(2 n+1)$-manifold if one can have the CR version of Laplacian comparison theorem. Indeed, this is the case for a $(2 n+1)$-dimensional Heisenberg group $H^{n}$ (see section 5 for details). Then we have

THEOREM 1.4. If $u(x, t)$ be a positive smooth solution of (1.6)

$$
\left(\Delta_{b}-\frac{\partial}{\partial t}\right) u(x, t)=0
$$

on $H^{n} \times[0, T)$ with

$$
P_{\beta} u=0
$$

at $t=0$, then $u$ satisfies the subgradient estimate

$$
t\left|\nabla_{b} \log u\right|^{2} \leq \frac{(n+2)\left(n^{2}+5 n+2\right)}{4(n+1)}+\epsilon
$$

on $H^{n} \times[0, T)$ for any $\epsilon>0$.

Remark 1.2. For the CR Yamabe flow on a closed pseudohermitian 3-manifold of zero torsion and nonnegative Tanaka-Webster curvature, we have the similar result on CR version of Li-Yau-Hamilton inequality ([CCW]).

As a consequence, we have the following Liouville-type theorems for CR heat equation on $H^{n} \times[0, \infty)$.

Corollary 1.5. Let $\left(H^{n}, J, \theta\right)$ be the standard $(2 n+1)$-dimensional Heisenberg group. If $u(x, t)$ is a positive solution of (1.6) on $H^{n} \times[0, \infty)$ with a positive smooth CR-pluriharmonic function as an initial. Then $u$ is a constant.

REMARK 1.3. It is true that there are no nontrivial positive harmonic functions on $H^{n}$. See $[K S]$ for details.

Now for any $L^{2}$-function $u(x, t)$, we may write

$$
u(x, t)=u_{\mathrm{ker}}(x, t)+u^{\perp}(x, t)
$$

with $P_{0}\left(u_{\text {ker }}(x, t)\right)=0$. From Lemma 2.2, we may split the CR heat equation (1.6) into the following heat equations respectively :

$$
\frac{\partial}{\partial t} u^{\perp}=\Delta_{b} u^{\perp}
$$

and

$$
\frac{\partial}{\partial t} u_{\mathrm{ker}}=\Delta_{b} u_{\mathrm{ker}}
$$

on Heisenberg group $\left(H^{n}, J, \theta\right)$. Observe that $H(x, y, t) \in C^{\infty}\left(H^{n} \times H^{n} \times \mathbf{R}^{+}\right)$and for any fixed $y, t, H(x, y, t) \in L^{2}\left(H^{n}\right)$. Then for any $L^{2}$-function $u(x, 0)=f(x)$, we have

$$
f(x)=f_{\text {ker }}(x)+f^{\perp}(x)
$$


and

$$
H(x, y, t)=H_{\mathrm{ker}}(x, y, t)+H^{\perp}(x, y, t)
$$

with $P_{0}\left(f_{\mathrm{ker}}(x)\right)=0$ and $P_{0}\left(H_{\mathrm{ker}}(x, y, t)\right)=0$. Hence

$$
u^{\perp}(y, t)=\int H^{\perp}(x, y, t) f^{\perp}(x) d x
$$

and

$$
u_{\mathrm{ker}}(y, t)=\int H_{\mathrm{ker}}(x, y, t) f_{\mathrm{ker}}(x) d x .
$$

As a consequence from Theorem 1.4 and Corollary 4.4, we have the following subgradient estimate of the heat kernel.

Corollary 1.6. Let $H(x, y, t)$ be the heat kernel of (1.6) on $H^{n} \times[0, T)$ with $H(x, y, t)=H_{\mathrm{ker}}(x, y, t)+H^{\perp}(x, y, t)$. Then for some constant $\delta$ and $0<\epsilon<1$,

$$
\left|\nabla_{b} H_{\mathrm{ker}}(x, y, t)\right| \leq C(\epsilon)^{\frac{\delta}{2}} t^{-\frac{(2 n+3)}{2}} \exp \left(-\frac{d_{c}^{2}(x, y)}{2(4+\epsilon) t}\right)
$$

with $C(\epsilon) \rightarrow \infty$ as $\epsilon \rightarrow 0$.

For simplicity, we first prove Theorems of this paper on a pseudohermitian $(2 n+$ 1)-manifold $(M, J, \theta)$ with $n=1$ as in section 3,4 . The higher dimensional cases will be given in section 5,6 .

Acknowledgments. The first named author would like to express his thanks to Prof. S.-T. Yau for constant encouragement, Prof. J.-P. Wang for valuable discussions during his visit at NCTS, Hsinchu, Taiwan. The first and second named authors would like to express their thanks to Prof. Jih-Hsin Cheng for his supports and Institute of Mathematics, Academia Sinica for the hospitality during the visit. Also the authors would like to express their thanks to the referee for valuable comments.

2. CR Bochner formula and preserving property. In this section, we will drive the $\mathrm{CR}$ version of Bochner formula and the preserving property for (1.6) on a pseudohermitian $\left(M^{2 n+1}, J, \theta\right)$.

We first derive the following $\mathrm{CR}$ version of Bochner formula on a complete pseudohermitian $\left(M^{2 n+1}, J, \theta\right)$.

LEMma 2.1. Let $\left(M^{2 n+1}, J, \theta\right)$ be a complete pseudohermitian manifold. For a real smooth function $u$ on $(M, J, \theta)$,

$$
\begin{aligned}
\frac{1}{2} \Delta_{b}\left|\nabla_{b} u\right|^{2}= & \left|\left(\nabla^{H}\right)^{2} u\right|^{2}+\left(1+\frac{2}{n}\right)<\nabla_{b} u, \nabla_{b} \Delta_{b} u>_{L_{\theta}} \\
& +[2 \text { Ric }-(n+2) \text { Tor }]\left(\left(\nabla_{b} u\right)_{\mathbf{C}},\left(\nabla_{b} u\right)_{\mathbf{C}}\right) \\
& -\frac{4}{n}<P u+\bar{P} u, d_{b} u>_{L_{\theta}^{*}} .
\end{aligned}
$$

Here $\left(\nabla_{b} u\right)_{\mathbf{C}}=u_{\bar{\alpha}} Z_{\alpha}$ is the corresponding complex (1,0)-vector field of $\nabla_{b} u$ and $d_{b} u=u_{\alpha} \theta^{\alpha}+u_{\bar{\alpha}} \theta^{\bar{\alpha}}$.

REMARK 2.1. In [Chi] and [CC], the CR Bochner formulae (2.1) was derived for $n=1$. 
Proof. First from $[\mathrm{Gr}]$, we have for a real function $u$

$$
\begin{aligned}
\frac{1}{2} \Delta_{b}\left|\nabla_{b} u\right|^{2}= & \left|\left(\nabla^{H}\right)^{2} u\right|^{2}+<\nabla_{b} u, \nabla_{b} \Delta_{b} u>_{L_{\theta}} \\
& +(2 \text { Ric }-n \text { Tor })\left(\left(\nabla_{b} u\right)_{\mathbf{C}},\left(\nabla_{b} u\right)_{\mathbf{C}}\right) \\
& -2 i \sum_{\alpha=1}^{n}\left(u_{\alpha} u_{\bar{\alpha} 0}-u_{\bar{\alpha}} u_{\alpha 0}\right) .
\end{aligned}
$$

We use the matrix $h_{\alpha \bar{\beta}}$ to raise and lower indices. In the following we always compute at one point. Then one may assume $h_{\alpha \bar{\beta}}=\delta_{\alpha \bar{\beta}}$ to lower the index. For instance,

$$
P_{\beta} \varphi=\sum_{\alpha=1}^{n}\left(\varphi \bar{\alpha} \alpha \beta+i n A_{\beta \alpha} \varphi \bar{\alpha}\right)
$$

and

$$
u_{0 \bar{\alpha}}-u_{\bar{\alpha} 0}=\sum_{\gamma=1}^{n} A_{\bar{\gamma}} \bar{\alpha} u_{\gamma}
$$

and

$$
i u_{0}=u_{\gamma \bar{\beta}}-u_{\bar{\beta} \gamma} .
$$

Compute

$$
\begin{aligned}
& i u_{\alpha} u_{\bar{\alpha} 0} \\
& =i u_{\alpha} u_{0 \bar{\alpha}}-i \sum_{\gamma=1}^{n} A \bar{\gamma} \bar{\alpha} u_{\alpha} u_{\gamma} \\
& =\frac{1}{n} u_{\alpha} \sum_{\beta=1}^{n}\left(u_{\beta \bar{\beta} \bar{\alpha}}-u_{\bar{\beta} \beta \bar{\alpha}}\right)-i \sum_{\gamma=1}^{n} A \bar{\gamma} \bar{\alpha} u_{\gamma} u_{\alpha} \\
& =\frac{1}{n} u_{\alpha} \bar{P}_{\alpha} u+i \sum_{\gamma=1}^{n} A \bar{\gamma} \bar{\alpha} u_{\alpha} u_{\gamma}-\frac{1}{n} \sum_{\beta=1}^{n}\left(u_{\alpha} u_{\bar{\beta} \beta \bar{\alpha}}\right) \\
& -i \sum_{\gamma=1}^{n} A \bar{\gamma} \bar{\alpha} u_{\gamma} u_{\alpha} \\
& =\frac{1}{n} u_{\alpha} \bar{P}_{\alpha} u-\frac{1}{n} \sum_{\beta=1}^{n}\left(u_{\alpha} u_{\bar{\beta} \beta \bar{\alpha}}\right)
\end{aligned}
$$

and

$$
-i u_{\bar{\alpha}} u_{\alpha 0}=\operatorname{conj}\left(i u_{\alpha} u_{\bar{\alpha} 0}\right) .
$$

Then

$$
\begin{aligned}
-2 i \sum_{\alpha=1}^{n}\left(u_{\alpha} u \bar{\alpha} 0-u \bar{\alpha} u_{\alpha 0}\right)= & -\frac{2}{n}<P u+\bar{P} u, d_{b} u>_{L_{\theta}^{*}} \\
& +\frac{2}{n} \sum_{\alpha, \beta=1}^{n}\left(u_{\alpha} u_{\bar{\beta} \beta \bar{\alpha}}+u_{\bar{\alpha}} u_{\beta \bar{\beta} \alpha}\right) .
\end{aligned}
$$

But

$$
\begin{aligned}
& <\nabla_{b} u, \nabla_{b} \Delta_{b} u>_{L_{\theta}} \\
& =\sum_{\alpha, \beta=1}^{n}\left[u_{\alpha}\left(u_{\bar{\beta} \beta}+u_{\beta \bar{\beta}}\right)_{\bar{\alpha}}+u_{\bar{\alpha}}\left(u_{\bar{\beta} \beta}+u_{\beta \bar{\beta}}\right)_{\alpha}\right] \\
& =\sum_{\alpha, \beta=1}^{n}\left(u_{\alpha} u_{\bar{\beta} \beta \bar{\alpha}}+u_{\bar{\alpha}} u_{\beta \bar{\beta} \alpha}\right)+\sum_{\alpha, \beta=1}^{n}\left(u_{\alpha} u_{\beta \bar{\beta} \bar{\alpha}}+u_{\bar{\alpha}} u_{\bar{\beta} \beta \alpha}\right) \\
& =\sum_{\alpha, \beta=1}^{n}\left(u_{\alpha} u_{\bar{\beta} \beta \bar{\alpha}}+u_{\bar{\alpha}} u_{\beta \bar{\beta} \alpha}\right)+<P u+\bar{P} u, d_{b} u>_{L_{\theta}^{*}} \\
& +i n \sum_{\gamma, \alpha=1}^{n}\left(A A_{\bar{\gamma}} u_{\alpha} u_{\gamma}-A_{\gamma \alpha} u_{\bar{\alpha}} u \bar{\gamma}\right) \\
& =\sum_{\alpha, \beta=1}^{n}\left(u_{\alpha} u_{\bar{\beta} \beta \bar{\alpha}}+u_{\bar{\alpha}} u_{\beta \bar{\beta} \alpha}\right)+<P u+\bar{P} u, d_{b} u>_{L_{\theta}^{*}} \\
& +n \operatorname{Tor}\left(\left(\nabla_{b} u\right)_{\mathbf{C}},\left(\nabla_{b} u\right)_{\mathbf{C}}\right) .
\end{aligned}
$$

It follows that

$$
\begin{aligned}
-2 i \sum_{\alpha=1}^{n}\left(u_{\alpha} u_{\bar{\alpha} 0}-u_{\bar{\alpha}} u_{\alpha 0}\right)= & -\frac{4}{n}<P u+\bar{P} u, d_{b} u>_{L_{\theta}^{*}} \\
& -2 \operatorname{Tor}\left(\left(\nabla_{b} u\right)_{\mathbf{C}},\left(\nabla_{b} u\right)_{\mathbf{C}}\right) \\
& +\frac{2}{n}<\nabla_{b} u, \nabla_{b} \Delta_{b} u>_{L_{\theta}} .
\end{aligned}
$$


Finally from (2.2) and (2.3), we have

$$
\begin{aligned}
\frac{1}{2} \Delta_{b}\left|\nabla_{b} u\right|^{2}= & \left|\left(\nabla^{H}\right)^{2} u\right|^{2}+\left(1+\frac{2}{n}\right)<\nabla_{b} u, \nabla_{b} \Delta_{b} u>_{L_{\theta}} \\
& +[2 \text { Ric }-(n+2) \text { Tor }]\left(\left(\nabla_{b} u\right)_{\mathbf{C}},\left(\nabla_{b} u\right)_{\mathbf{C}}\right) \\
& -\frac{4}{n}<P u+\bar{P} u, d_{b} u>_{L_{\theta}^{*}} .
\end{aligned}
$$

Lemma 2.2. Let $(M, J, \theta)$ be a closed pseudohermitian $(2 n+1)$-manifold of zero torsion. If $u(x, t)$ is a solution of

$$
\left(\Delta_{b}-\frac{\partial}{\partial t}\right) u(x, t)=0
$$

on $M \times[0, \infty)$ with $P_{\beta} u(x, 0)=0$. Then $P_{\beta} u(x, t)=0$ for all $t \in(0, \infty)$.

Proof. Let $(M, J, \theta)$ be a closed pseudohermitian $(2 n+1)$-manifold of zero torsion. From Remark 1.1, we have $P_{0} u=0$ if and only if $P_{\beta} u=0$ and

$$
P_{0} u=\left(\left(\Delta_{b}\right)^{2} u+n T^{2} u\right) .
$$

It follows that $\Delta_{b} P_{0} u=P_{0} \Delta_{b} u$. Apply $P_{0}$ to the heat equation, we obtain

$$
\left(\Delta_{b}-\frac{\partial}{\partial t}\right) P_{0} u(x, t)=0
$$

on $M \times[0, \infty)$ with $P_{0} u(x, 0)=0$. Hence the Lemma follows from the maximum principle and Remark 1.1. $\square$

Lemma 2.3. Let $\left(H^{n}, J, \theta\right)$ be the standard $(2 n+1)$-dimensional Heisenberg group. If $u(x, t)$ is a solution of

$$
\left(\Delta_{b}-\frac{\partial}{\partial t}\right) u(x, t)=0
$$

on $M \times[0, \infty)$ with $P_{\beta} u(x, 0)=0, \beta=1, \ldots, n$. Then $P_{\beta} u(x, t)=0$ for all $t \in[0, \infty)$.

REMARK 2.2. Since $\left(H^{n}, J, \theta\right)$ is complete noncompact, $P_{\beta} u$ is not necessarily vanishing even if $P_{0} u=0$. So we need to have a different proof from Lemma 2.2.

Proof. We first do it for $n=1$. We need the following commutation relation ([L1])

$$
\begin{aligned}
& C_{I, 01}-C_{I, 10}=C_{I, \overline{1}} A_{11}-k C_{I} A_{11, \overline{1}}, \\
& C_{I, 0 \overline{1}}-C_{I, \overline{1} 0}=C_{I, 1} A_{\overline{11}}+k C_{I} A_{\overline{11}, 1}, \\
& C_{I, 1 \overline{1}}-C_{I, \overline{1} 1}=i C_{I, 0}+k W C_{I},
\end{aligned}
$$

Here $C_{I}$ denotes a coefficient of a tensor with multi-index $I$ consisting of only 1 and $\overline{1}$, and $k$ is the number of 1 minus the number of $\overline{1}$ in $I$.

For

$$
\begin{aligned}
\frac{\partial}{\partial t} u_{\overline{1} 11} & =\left(\Delta_{b} u\right)_{\overline{111}} \\
& =\left(u_{\overline{1} 1}+u_{1 \overline{1}}\right)_{\overline{1} 11} \\
& =\left(u_{\overline{1} 1 \overline{1} 11}+u_{\overline{1 \overline{1}}_{11}}\right),
\end{aligned}
$$


it follows from (2.4) that

$$
u_{\overline{1} 1 \overline{1} 1}=u_{\overline{1} 11 \overline{1} 1}-i u_{\overline{1} 101}=u_{\overline{1} 11 \overline{1} 1}-i u_{\overline{1} 110}
$$

and

$$
\begin{aligned}
u_{1 \overline{11} 11} & =u_{\overline{1}_{1111}}+i u_{\mathrm{o} \overline{1} 11} \\
& =\left(u_{\bar{T}_{11 \overline{1} 1}}-i u_{\overline{1} 101}\right)+i u_{\mathrm{oT} 11} \\
& =\left(u_{\overline{1}_{111 \overline{1}}}-i u_{\overline{1}_{110}}\right)-i u_{\overline{1} 101}+i u_{\overline{o \overline{1}}_{11}} \\
& =u_{\overline{\overline{1}}_{111 \overline{1}}}-i u_{\overline{\overline{1}}_{110}} .
\end{aligned}
$$

Thus for $\mathcal{L}_{2}=-\Delta_{b}+2 i T$

$$
\frac{\partial}{\partial t} u_{\overline{1} 11}=\Delta_{b} u_{\overline{1} 11}-2 i u_{\overline{1} 110}=-\mathcal{L}_{2} u_{\overline{1} 11} .
$$

This plus (2.5) imply

$$
\frac{\partial}{\partial t}\left(P_{1} u\right)=-\mathcal{L}_{2}\left(P_{1} u\right) .
$$

Similarly for $n \geq 2$, we have

$$
\frac{\partial}{\partial t}\left(P_{\beta} u\right)=\frac{\partial}{\partial t}\left(\sum_{\alpha=1}^{n} u_{\bar{\alpha} \alpha \beta}\right)=\sum_{\alpha=1}^{n}\left(\Delta_{b} u\right)_{\bar{\alpha} \alpha \beta}=\sum_{\gamma, \alpha=1}^{n}\left(u_{\gamma \bar{\gamma}}+u_{\bar{\gamma} \gamma}\right) \bar{\alpha} \alpha \beta .
$$

Now by commutation relations

$$
\begin{aligned}
u_{\gamma \overline{\gamma \alpha} \alpha \beta} & =u_{\bar{\gamma} \gamma \bar{\alpha} \alpha \beta}+i u_{0 \bar{\alpha} \alpha \beta} \\
& =u_{\bar{\gamma} \gamma \bar{\alpha} \beta \alpha}+i u_{\bar{\alpha} \alpha \beta 0} \\
& =u_{\bar{\gamma} \gamma \beta \bar{\alpha} \alpha}-i u_{\bar{\gamma} \gamma 0 \alpha}+i u_{\bar{\alpha} \alpha \beta 0} \\
& =u_{\bar{\gamma} \gamma \beta \bar{\alpha} \alpha}-i u_{\bar{\gamma} \gamma \alpha 0}+i u_{\bar{\alpha} \alpha \beta 0}
\end{aligned}
$$

and

$$
\begin{aligned}
u_{\bar{\gamma} \gamma \bar{\alpha} \alpha \beta} & =u_{\bar{\gamma} \gamma \bar{\alpha} \beta \alpha} \\
& =u_{\bar{\gamma} \gamma \beta \bar{\alpha} \alpha}-i u_{\bar{\gamma} \gamma 0 \alpha} \\
& =u_{\bar{\gamma} \gamma \beta \alpha \bar{\alpha}}-i u_{\bar{\gamma} \gamma \beta 0}-i u_{\bar{\gamma} \gamma \alpha 0} .
\end{aligned}
$$

It follows from (2.6) and (2.7) that

$$
\begin{aligned}
\frac{\partial}{\partial t}\left(P_{\beta} u\right) & =\sum_{\gamma, \alpha=1}^{n}\left(u_{\gamma \overline{\gamma \alpha} \alpha \beta}+u_{\bar{\gamma} \gamma \bar{\alpha} \alpha \beta}\right) \\
& =\Delta_{b}\left(P_{\beta} u\right)-2 i \sum_{\gamma, \alpha=1}^{n} u_{\bar{\gamma} \gamma \alpha 0}+i \sum_{\gamma, \alpha=1}^{n} u u_{\alpha} \alpha \beta 0-i \sum_{\gamma, \alpha=1}^{n} u_{\bar{\gamma} \gamma \beta 0} \\
& =\Delta_{b}\left(P_{\beta} u\right)-2 i \sum_{\gamma, \alpha=1}^{n} u_{\bar{\gamma} \gamma \alpha 0} \\
& =\Delta_{b}\left(P_{\beta} u\right)-2 i T\left(\sum_{\alpha=1}^{n} P_{\alpha} u\right) .
\end{aligned}
$$

Hence

$$
\frac{\partial}{\partial t}\left(\sum_{\beta=1}^{n} P_{\beta} u\right)=\Delta_{b}\left(\sum_{\beta=1}^{n} P_{\beta} u\right)-i 2 n T\left(\sum_{\beta=1}^{n} P_{\beta} u\right) .
$$

That is

$$
\frac{\partial}{\partial t}\left(\sum_{\beta=1}^{n} P_{\beta} u\right)=-\mathcal{L}_{2 n}\left(\sum_{\beta=1}^{n} P_{\beta} u\right) .
$$

Here $\mathcal{L}_{2 n}=-\Delta_{b}+i 2 n T$. Since $2 n$ is not an odd integer, $-\mathcal{L}_{2 n}$ is a subelliptic operator again. Then by the uniqueness of solution to subelliptic parabolic equation, $P_{\beta} u(x, t)=0$ for all $t \in[0, \infty)$ if $P_{\beta} u(x, 0)=0, \quad \beta=1, \ldots, n$. $\square$ 
3. Subgradient estimate of the CR heat equation. In this section, we first establish the subgradient estimate of Theorem 1.3 for $n=1$. For $n \geq 2$, we refer it to section 6 .

Let $(M, J, \theta)$ be a closed pseudohermitian 3-manifold. By using the arguments of $[\mathrm{LY}]$, we are able to derive the $\mathrm{CR}$ version of parabolic Li-Yau gradient estimate for the positive solution $u(x, t)$ of $(1.6)$ on $M \times[0, \infty)$.

Let $\varphi=\log u$. Then $\varphi$ satisfies

$$
\left(\Delta_{b}-\frac{\partial}{\partial t}\right) \varphi=-\left|\nabla_{b} \varphi\right|^{2} .
$$

On the other hand, from Cao-Yau's $([\mathrm{CY}])$ paper, one has the standard parabolic Li-Yau gradient estimate.

Proposition 3.1. ([CY, Theorem 2.1]) Let $(M, J, \theta)$ be a closed pseudohermitian $(2 n+1)$-manifold and $u(x, t)$ be a positive smooth solution of $(1.6)$ on $M \times[0, \infty)$. Then there exist constants $C^{\prime}, C^{\prime \prime}$ and $\delta_{0}>1$ such that for any $\delta \geq \delta_{0}$, u satisfies the estimate

$$
\frac{\left|\nabla_{b} u\right|^{2}}{u^{2}}-\delta \frac{u_{t}}{u} \leq \frac{C^{\prime}}{t}+C^{\prime \prime}
$$

on $M \times[0, \infty)$.

Now we derive the CR version of parabolic Li-Yau gradient estimate for the positive solution of the $\mathrm{CR}$ heat equation. First, we need the following Lemma.

LEMma 3.2. Let $(M, J, \theta)$ be a closed pseudohermitian $(2 n+1)$-manifold. Let $\varphi=\ln f$, for $f>0$. Then

$$
\begin{aligned}
& \left\langle P \varphi+\bar{P} \varphi, d_{b} \varphi\right\rangle_{L_{\theta}^{*}} \\
& =f^{-2}\left\langle P f+\bar{P} f, d_{b} f\right\rangle_{L_{\theta}^{*}}-\frac{1}{2}\left\langle\nabla_{b} \varphi, \nabla_{b}\left|\nabla_{b} \varphi\right|^{2}\right\rangle-\frac{1}{2} f^{-1} \Delta_{b} f\left|\nabla_{b} \varphi\right|^{2} .
\end{aligned}
$$

Proof. In the following, we use the Einstein convention notation. Let $Q(x)=$ $\left|\nabla_{b} \varphi\right|^{2}(x)$. We compute

$$
\begin{aligned}
\nabla_{b} Q & =Q_{\bar{\alpha}} Z_{\alpha}+Q_{\alpha} Z_{\bar{\alpha}}=2 \nabla_{b}\left(\varphi_{\alpha} \varphi_{\bar{\alpha}}\right) \\
& =2 f^{-4}\left(f^{2} f_{\alpha} f_{\bar{\alpha} \bar{\beta}}+f^{2} f_{\bar{\alpha}} f_{\alpha \bar{\beta}}-2 f_{\alpha} f_{\bar{\alpha}} f_{\bar{\beta}}\right) Z_{\beta}+\text { complex conjugate. }
\end{aligned}
$$

It follows that

$$
\begin{aligned}
& P_{\beta} \varphi \\
= & \varphi \bar{\alpha} \alpha \beta+i n A_{\beta \alpha} \varphi \bar{\alpha} \\
= & f^{-4}\left(f^{3} f_{\bar{\alpha} \alpha \beta}-f^{2} f_{\alpha} f_{\bar{\alpha} \beta}-f^{2} f_{\bar{\alpha}} f_{\alpha \beta}-f^{2} f_{\beta} f_{\bar{\alpha} \alpha}+2 f f_{\alpha} f_{\beta} f_{\bar{\alpha}}\right)+i n A_{\beta \alpha} f^{-1} f_{\bar{\alpha}} \\
= & f^{-1}\left(P_{\beta} f-\frac{1}{2} f Q_{\beta}-f^{-1} f_{\beta} f_{\bar{\alpha} \alpha}\right) \\
= & f^{-1}\left(P_{\beta} f-\frac{1}{2} f Q_{\beta}-\varphi_{\beta} f_{\bar{\alpha} \alpha}\right),
\end{aligned}
$$


thus

$$
\begin{aligned}
\left\langle P \varphi+\bar{P} \varphi, d_{b} \varphi\right\rangle_{L_{\theta}^{*}} & =\left\langle\left(P_{\beta} \varphi\right) \theta^{\beta}+\left(\bar{P}_{\beta} \varphi\right) \theta^{\bar{\beta}}, \varphi_{\beta} \theta^{\beta}+\varphi_{\bar{\beta}} \theta^{\bar{\beta}}\right\rangle_{L_{\theta}^{*}} \\
& =\left(P_{\beta} \varphi\right) \varphi_{\bar{\beta}}+\left(\bar{P}_{\beta} \varphi\right) \varphi_{\beta} \\
& =f^{-1}\left(P_{\beta} f-\frac{1}{2} f Q_{\beta}-\varphi_{\beta} f_{\bar{\alpha} \alpha}\right) \varphi_{\bar{\beta}}+\text { complex conjugate } \\
& =f^{-2}\left\langle P f+\bar{P} f, d_{b} f\right\rangle_{L_{\theta}^{*}}-\frac{1}{2}\left\langle\nabla_{b} \varphi, \nabla_{b}\left|\nabla_{b} \varphi\right|^{2}\right\rangle-\frac{1}{2} f^{-1} \Delta_{b} f\left|\nabla_{b} \varphi\right|^{2}
\end{aligned}
$$

This implies the Lemma.

Lemma 3.3. Let $(M, J, \theta)$ be a closed pseudohermitian 3-manifold. If $u(x, t)$ is the positive smooth solution $u(x, t)$ of (1.6) on $M \times[0, \infty)$. Suppose that

$$
(2 \operatorname{Ric}-3 \text { Tor })(Z, Z) \geq-k_{0}|Z|^{2},
$$

for all $Z \in T_{1,0}$ and $k_{0}$ is a nonnegative constant. Then the function

$$
F=t\left(\left|\nabla_{b} \varphi\right|^{2}+3 \varphi_{t}\right)
$$

satisfies the inequality

$$
\begin{aligned}
\left(\Delta_{b}-\frac{\partial}{\partial t}\right) F \geq & -\frac{2}{3}\left\langle\nabla_{b} \varphi, \nabla_{b} F\right\rangle+\frac{1}{9 t} F(F-9)+ \\
& -k_{0} t\left|\nabla_{b} \varphi\right|^{2}-8 t u^{-2}\left\langle P u+\bar{P} u, d_{b} u\right\rangle_{L_{\theta}^{*}} .
\end{aligned}
$$

Proof. First differentiating (3.3) w.r.t. the $t$-variable, we have

$$
\begin{aligned}
F_{t} & =\frac{1}{t} F+t\left(\left|\nabla_{b} \varphi\right|^{2}+3 \varphi_{t}\right)_{t} \\
& =\frac{1}{t} F+t\left(4\left|\nabla_{b} \varphi\right|^{2}+3 \Delta_{b} \varphi\right)_{t} \\
& =\frac{1}{t} F+t\left[8\left\langle\nabla_{b} \varphi, \nabla_{b} \varphi_{t}\right\rangle+3 \Delta_{b} \varphi_{t}\right] .
\end{aligned}
$$

By using the CR version of Bochner formula (2.1) and Lemma 3.2, one obtains

$$
\begin{aligned}
\Delta_{b} F= & t\left(\Delta_{b}\left|\nabla_{b} \varphi\right|^{2}+3 \Delta_{b} \varphi_{t}\right) \\
= & t\left[2\left|\left(\nabla^{H}\right)^{2} \varphi\right|^{2}+6\left\langle\nabla_{b} \varphi, \nabla_{b} \Delta_{b} \varphi\right\rangle\right. \\
& +2(2 \operatorname{Ric}-3 \text { Tor })\left(\left(\nabla_{b} \varphi\right)_{\mathbf{C}},\left(\nabla_{b} \varphi\right)_{\mathbf{C}}\right) \\
& \left.-8\left\langle P \varphi+\bar{P} \varphi, d_{b} \varphi\right\rangle_{L_{\theta}^{*}}+3 \Delta_{b} \varphi_{t}\right] \\
\geq & t\left[4\left|\varphi_{11}\right|^{2}+\left(\Delta_{b} \varphi\right)^{2}+6\left\langle\nabla_{b} \varphi, \nabla_{b} \Delta_{b} \varphi\right\rangle-k_{0}\left|\nabla_{b} \varphi\right|^{2}\right. \\
& \left.-8\left\langle P \varphi+\bar{P} \varphi, d_{b} \varphi\right\rangle_{L_{\theta}^{*}}+3 \Delta_{b} \varphi_{t}\right] \\
= & t\left[4\left|\varphi_{11}\right|^{2}+\left(\Delta_{b} \varphi\right)^{2}+6\left\langle\nabla_{b} \varphi, \nabla_{b} \Delta_{b} \varphi\right\rangle-k_{0}\left|\nabla_{b} \varphi\right|^{2}\right. \\
& -8 u^{-2}\left\langle P u+\bar{P} u, d_{b} u\right\rangle_{L_{\theta}^{*}}+4 \varphi_{t}\left|\nabla_{b} \varphi\right|^{2} \\
& \left.+4\left\langle\nabla_{b} \varphi, \nabla_{b}\left|\nabla_{b} \varphi\right|^{2}\right\rangle+3 \Delta_{b} \varphi_{t}\right] .
\end{aligned}
$$


Here we have used the inequalities

$$
\begin{gathered}
\left|\left(\nabla^{H}\right)^{2} \varphi\right|^{2}=2\left|\varphi_{11}\right|^{2}+\frac{1}{2}\left(\Delta_{b} \varphi\right)^{2}+\frac{1}{2} \varphi_{0}^{2} \geq 2\left|\varphi_{11}\right|^{2}+\frac{1}{2}\left(\Delta_{b} \varphi\right)^{2}, \\
(2 \text { Ric }-3 \text { Tor })\left(\left(\nabla_{b} \varphi\right)_{\mathbb{C}},\left(\nabla_{b} \varphi\right)_{\mathbb{C}}\right) \geq-k_{0}\left|\left(\nabla_{b} \varphi\right)_{\mathbb{C}}\right|^{2}=-\frac{k_{0}}{2}\left|\nabla_{b} \varphi\right|^{2},
\end{gathered}
$$

and

$$
\varphi_{t}=\frac{u_{t}}{u}=\frac{\Delta_{b} u}{u}
$$

Applying the formula

$$
\Delta_{b} \varphi=\varphi_{t}-\left|\nabla_{b} \varphi\right|^{2}=\frac{1}{3 t} F-\frac{4}{3}\left|\nabla_{b} \varphi\right|^{2}
$$

and combining (3.4), (3.5), we conclude

$$
\begin{aligned}
\left(\Delta_{b}-\frac{\partial}{\partial t}\right) F \geq & -\frac{1}{t} F+t\left[4\left|\varphi_{11}\right|^{2}+\left(\Delta_{b} \varphi\right)^{2}+6\left\langle\nabla_{b} \varphi, \nabla_{b} \Delta_{b} \varphi\right\rangle\right. \\
& +4\left\langle\nabla_{b} \varphi, \nabla_{b}\left|\nabla_{b} \varphi\right|^{2}\right\rangle-8\left\langle\nabla_{b} \varphi, \nabla_{b} \varphi_{t}\right\rangle \\
& \left.-k_{0}\left|\nabla_{b} \varphi\right|^{2}+4 \varphi_{t}\left|\nabla_{b} \varphi\right|^{2}-8 u^{-2}\left\langle P u+\bar{P} u, d_{b} u\right\rangle_{L_{\theta}^{*}}\right] \\
= & -\frac{1}{t} F+t\left[-\frac{2}{3 t}\left\langle\nabla_{b} \varphi, \nabla_{b} F\right\rangle-\frac{4}{3}\left\langle\nabla_{b} \varphi, \nabla_{b}\left|\nabla_{b} \varphi\right|^{2}\right\rangle+4\left|\varphi_{11}\right|^{2}\right. \\
& \left.+\left(\Delta_{b} \varphi\right)^{2}-k_{0}\left|\nabla_{b} \varphi\right|^{2}+4 \varphi_{t}\left|\nabla_{b} \varphi\right|^{2}-8 u^{-2}\left\langle P u+\bar{P} u, d_{b} u\right\rangle_{L_{\theta}^{*}}\right] .
\end{aligned}
$$

Now it is easy to see that

$$
\left\langle\nabla_{b} \varphi, \nabla_{b}\left|\nabla_{b} \varphi\right|^{2}\right\rangle=4 \operatorname{Re}\left(\varphi_{11} \varphi_{\overline{1}} \varphi_{\overline{1}}\right)+\Delta_{b} \varphi\left|\nabla_{b} \varphi\right|^{2} .
$$

Thus

$$
\begin{aligned}
-\frac{4}{3}\left\langle\nabla_{b} \varphi, \nabla_{b}\left|\nabla_{b} \varphi\right|^{2}\right\rangle & =-\frac{16}{3} \operatorname{Re}\left(\varphi_{11} \varphi_{\overline{1}} \varphi_{\overline{1}}\right)-\frac{4}{3} \Delta_{b} \varphi\left|\nabla_{b} \varphi\right|^{2} \\
& \geq-4\left|\varphi_{11}\right|^{2}-\frac{16}{9}\left|\varphi_{\overline{1}}\right|^{4}-\frac{4}{3} \Delta_{b} \varphi\left|\nabla_{b} \varphi\right|^{2} \\
& =-4\left|\varphi_{11}\right|^{2}-\frac{4}{9}\left|\nabla_{b} \varphi\right|^{4}-\frac{4}{3} \Delta_{b} \varphi\left|\nabla_{b} \varphi\right|^{2} .
\end{aligned}
$$

Here we have used the basic inequality $2 \operatorname{Re}(z w) \leq \epsilon|z|^{2}+\epsilon^{-1}|w|^{2}$ for all $\epsilon>0$. All these imply

$$
\begin{aligned}
\left(\Delta_{b}-\frac{\partial}{\partial t}\right) F \geq & -\frac{1}{t} F-\frac{2}{3}\left\langle\nabla_{b} \varphi, \nabla_{b} F\right\rangle+t\left[\left(\Delta_{b} \varphi\right)^{2}+\frac{8}{3} \Delta_{b} \varphi\left|\nabla_{b} \varphi\right|^{2}\right. \\
& \left.+\frac{32}{9}\left|\nabla_{b} \varphi\right|^{4}-k_{0}\left|\nabla_{b} \varphi\right|^{2}-8 u^{-2}\left\langle P u+\bar{P} u, d_{b} u\right\rangle_{L_{\theta}^{*}}\right] \\
\geq & -\frac{2}{3}\left\langle\nabla_{b} \varphi, \nabla_{b} F\right\rangle+\frac{1}{9 t} F(F-9) \\
& -k_{0} t\left|\nabla_{b} \varphi\right|^{2}-8 u^{-2}\left\langle P u+\bar{P} u, d_{b} u\right\rangle_{L_{\theta}^{*}} .
\end{aligned}
$$

This completes the proof of Lemma 3.3.

TheOREm 3.4. Let $(M, J, \theta)$ be a closed pseudohermitian 3-manifold of zero torsion and nonnegative Tanaka-Webster scalar curvature. If $u(x, t)$ is the positive solution of (1.6) on $M \times[0, \infty)$ such that

$$
P_{1} u=0
$$


at $t=0$. Then $u$ satisfies the estimate

$$
\frac{\left|\nabla_{b} u\right|^{2}}{u^{2}}+3 \frac{u_{t}}{u} \leq \frac{9}{t}
$$

on $M \times[0, \infty)$.

Proof. Applying Lemma 3.3 to $\varphi$ by setting $A_{11}=0, k_{0}=0$ and

$$
\left\langle P u+\bar{P} u, d_{b} u\right\rangle=0 .
$$

Then we have

$$
\left(\Delta_{b}-\frac{\partial}{\partial t}\right) F \geq-\frac{2}{3}\left\langle\nabla_{b} \varphi, \nabla_{b} F\right\rangle+\frac{1}{9 t} F(F-9) .
$$

The theorem claims that $F$ is at most 9 . If not, at the maximum point $\left(x_{0}, t_{0}\right)$ of $F$ on $M \times[0, T]$ for some $T>0$,

$$
F\left(x_{0}, t_{0}\right)>9 .
$$

Clearly, $t_{0}>0$, because $F(x, 0)=0$. By the fact that $\left(x_{0}, t_{0}\right)$ is a maximum point of $F$ on $M \times[0, T]$, we have

$$
\begin{aligned}
& \Delta_{b} F\left(x_{0}, t_{0}\right) \leq 0, \\
& \nabla_{b} F\left(x_{0}, t_{0}\right)=0,
\end{aligned}
$$

and

$$
F_{t}\left(x_{0}, t_{0}\right) \geq 0 .
$$

Combining with (3.9), this implies

$$
0 \geq \frac{1}{9 t_{0}} F\left(x_{0}, t_{0}\right)\left(F\left(x_{0}, t_{0}\right)-9\right),
$$

which is a contradiction. Hence $F \leq 9$ and the theorem follows.

Then by combining Proposition 3.1 and Theorem 3.4, the subgradient estimate Theorem 1.3 follows easily for $n=1$.

4. Subgradient estimates in the Heisenberg group $H^{1}$. In this section, we first establish Liouville-type theorems for the CR heat equation on a 3-dimensional Heisenberg group $H^{1}$. Secondly, we derive the subgradient estimate for CR Heat Kernel on $H^{1}$.

From $[\mathrm{PP}]$, we recall the following result.

Proposition 4.1. If $u(x, t)$ be a positive smooth solution of (1.6) on $H^{n} \times[0, T)$, then $u$ satisfies the estimate

$$
\frac{\left|\nabla_{b} u\right|^{2}}{u^{2}}-\frac{u_{t}}{u} \leq \frac{n}{t}
$$

on $H^{n} \times[0, T)$. 
THEOREM 4.2. If $u(x, t)$ be a positive smooth solution of (1.6)

$$
\left(\Delta_{b}-\frac{\partial}{\partial t}\right) u(x, t)=0
$$

on $H^{1} \times[0, T)$ with

$$
P_{1} u=0
$$

at $t=0$, then $u$ satisfies the subgradient estimate

$$
\frac{\left|\nabla_{b} u\right|^{2}}{u^{2}}+3 \frac{u_{t}}{u} \leq \frac{9+\epsilon}{t}
$$

on $H^{1} \times[0, T)$ for any $\epsilon>0$.

Proof. Let $B_{2 R}$ be a ball of radius $2 R$ center at $O \in H^{1}$. Let $\varphi=\log u$ and $F=t\left(\left|\nabla_{b} \varphi\right|^{2}+3 \varphi_{t}\right)$, then

$$
\sup _{B_{R}}\left(\frac{\left|\nabla_{b} u\right|^{2}}{u^{2}}+3 \frac{u_{t}}{u}\right)=\sup _{B_{R}} \frac{F}{t} .
$$

Let $\psi \in C_{0}^{\infty}(R)$ be a cut-off function ([DT]) such that $0 \leq \psi \leq 1, \psi(t) \equiv 1$ for $t \in[0,1], \psi(t) \equiv 0$ for $t \geq 2$. We also require

$$
\psi^{\prime} \leq 0, \quad \psi^{\prime \prime} \geq-C_{1}, \quad \text { and } \frac{\left|\psi^{\prime}\right|^{2}}{\psi} \leq C_{2},
$$

where $C_{1}$ and $C_{2}$ are positive constants. Denote by $d_{c}(x)$ be the Carnot-Carathéodory distance from $O$ to $x$ in $H^{1}$. Then we define $\eta(x)=\psi\left(\frac{d_{c}(x)}{R}\right)$. It is clear that supp $\eta \subset B_{2 R}$ and $\left.\eta\right|_{B_{R}} \equiv 1$.

We want to apply the maximum principle to $\eta F$. The function $\eta$ may not be smooth at the cut locus of $O \in H^{1}$. However, when applying the maximum principle, we can assume $\eta$ is differentiable as in [LY].

If $\eta F$ attains its maximum at $\left(x_{0}, t_{0}\right) \in B_{2 R} \times\left[0, T^{\prime}\right]$ with $0<T^{\prime}<T$, clearly we may assume $(\eta F)\left(x_{0}, t_{0}\right)>0$ (otherwise $F \leq 0$, and the theorem is true). So $x_{0} \in B_{2 R}, t_{0}>0$, and by the maximum principle, at $\left(x_{0}, t_{0}\right)$

$$
\begin{gathered}
\nabla_{b}(\eta F)=F \nabla_{b} \eta+\eta \nabla_{b} F=0 \\
\Delta_{b}(\eta F) \leq 0
\end{gathered}
$$

and

$$
\frac{\partial}{\partial t}(\eta F)=\eta F_{t} \geq 0
$$

In the sequel, all computations will be at the point $\left(x_{0}, t_{0}\right)$. By (4.2), $\nabla_{b} F=$ $-F \nabla_{b} \eta / \eta$, and by $(4.3)$

$$
\begin{aligned}
0 & \geq \Delta_{b}(\eta F)=F \Delta_{b} \eta+\eta \Delta_{b} F+2\left\langle\nabla_{b} \eta, \nabla_{b} F\right\rangle \\
& =F \Delta_{b} \eta+\eta \Delta_{b} F-2 F \frac{\left|\nabla_{b} \eta\right|^{2}}{\eta} .
\end{aligned}
$$


By (4.1), we have

$$
\frac{\left|\nabla_{b} \eta\right|^{2}}{\eta}=\frac{\left|\psi^{\prime}\right|^{2}\left|\nabla_{b} d_{c}\right|^{2}}{R^{2} \psi}=\frac{\left|\psi^{\prime}\right|^{2}}{R^{2} \psi} \leq \frac{C_{2}}{R^{2}}
$$

and

$$
\Delta_{b} \eta=\frac{\psi^{\prime \prime}\left|\nabla_{b} d_{c}\right|^{2}}{R^{2}}+\frac{\psi^{\prime} \Delta_{b} d_{c}}{R}=\frac{\psi^{\prime \prime}}{R^{2}}+\frac{\psi^{\prime}}{R} \Delta_{b} d_{c} \geq-\frac{C_{1}}{R^{2}}-\frac{\sqrt{C_{2}}}{R} \Delta_{b} d_{c}
$$

Since in $H^{1}$, we have the sub-Laplacian comparison $(*)$ (see the proof in next section)

$$
\Delta_{b} d_{c} \leq \frac{C}{d_{c}},
$$

for some constant $C$. Then

$$
\Delta_{b} \eta \geq-\frac{C_{3}}{R^{2}}
$$

Substituting this into (4.5) and applying Lemma 2.3 and Lemma 3.3 with $A_{11}=0$, $k_{0}=0$, all these imply

$$
\begin{aligned}
0 & \geq \Delta_{b}(\eta F) \geq-\frac{C_{3}}{R^{2}} F-2 F \frac{\left|\nabla_{b} \eta\right|^{2}}{\eta}+\eta \Delta_{b} F \\
& \geq-\frac{C_{3}}{R^{2}} F-2 F \frac{\left|\nabla_{b} \eta\right|^{2}}{\eta}+\eta\left[F_{t}+2\left\langle\nabla_{b} \varphi, \nabla_{b} F\right\rangle+\frac{1}{9 t} F(F-9)\right] .
\end{aligned}
$$

Since $\eta F_{t}=(\eta F)_{t} \geq 0,2 \eta\left\langle\nabla_{b} \varphi, \nabla_{b} F\right\rangle=\frac{2}{3} F\left\langle\nabla_{b} \varphi, \nabla_{b} \eta\right\rangle$, the above inequality can be reduced as

$$
0 \geq-\frac{C_{3}}{R^{2}} F-2 F \frac{\left|\nabla_{b} \eta\right|^{2}}{\eta}+\frac{2}{3} F\left\langle\nabla_{b} \varphi, \nabla_{b} \eta\right\rangle+\frac{1}{9 t} \eta F(F-9),
$$

and multiplying by $\eta$, we get

$$
\begin{aligned}
0 & \geq-\frac{C_{3}}{R^{2}} \eta F-2 F\left|\nabla_{b} \eta\right|^{2}+\frac{2}{3} F \eta\left\langle\nabla_{b} \varphi, \nabla_{b} \eta\right\rangle+\frac{1}{9 t} \eta^{2} F(F-9) \\
& =(\eta F)\left(-\frac{C_{3}}{R^{2}}-2 \frac{\left|\nabla_{b} \eta\right|^{2}}{\eta}-\frac{\eta}{t}\right)+\frac{2}{3} \eta F\left\langle\nabla_{b} \varphi, \nabla_{b} \eta\right\rangle+\frac{1}{9 t}(\eta F)^{2} \\
& \geq(\eta F)\left(-\frac{C_{3}}{R^{2}}-2 \frac{\left|\nabla_{b} \eta\right|^{2}}{\eta}-\frac{\eta}{t}\right)-2 \eta F\left|\nabla_{b} \varphi\right|\left|\nabla_{b} \eta\right|+\frac{1}{9 t}(\eta F)^{2}
\end{aligned}
$$

Using $0 \leq \eta \leq 1$, and (4.6), we get

$$
\begin{aligned}
0 & \geq(\eta F)\left(-\frac{C_{3}}{R^{2}}-2 \frac{C_{2}}{R^{2}}-\frac{1}{t}\right)-2 \eta^{3 / 2} F \frac{\sqrt{C_{2}}}{R}\left|\nabla_{b} \varphi\right|+\frac{1}{9 t}(\eta F)^{2} \\
& =(\eta F)\left(-\frac{1}{t}-\frac{C_{4}}{R^{2}}\right)-2 \eta^{3 / 2} F \frac{\sqrt{C_{2}}}{R}\left|\nabla_{b} \varphi\right|+\frac{1}{9 t}(\eta F)^{2},
\end{aligned}
$$

where $C_{4}=C_{3}+2 C_{2}$. Multiplying by $t$ to the above inequality, this leads to

$$
\begin{aligned}
0 & \geq(\eta F)\left(\frac{1}{9} \eta F-1-\frac{C_{4}}{R^{2}} t\right)-2 t \eta^{3 / 2} F \frac{\sqrt{C_{2}}}{R}\left|\nabla_{b} \varphi\right| \\
& =(\eta F)\left(\frac{1}{9} \eta F-1-\frac{C_{4}}{R^{2}} t-\frac{2 \sqrt{C_{2}}}{R} \eta^{1 / 2}\left|\nabla_{b} \varphi\right| t\right) .
\end{aligned}
$$


Therefore, we get

$$
\eta F \leq 9+\frac{9 C_{4}}{R^{2}} t+\frac{18 \sqrt{C_{2}}}{R} \eta^{1 / 2}\left|\nabla_{b} \varphi\right| t .
$$

(i) If $\varphi_{t}\left(x_{0}, t_{0}\right)<0$, then, by the Proposition $4.1,\left|\nabla_{b} \varphi\right|^{2} \leq\left|\nabla_{b} \varphi\right|^{2}-\varphi_{t} \leq 1 / t$ and using $0 \leq \eta \leq 1$, we have

$$
\eta F \leq 9+\frac{C_{4}}{R^{2}} t+\frac{18 \sqrt{C_{2}}}{R} t^{1 / 2} .
$$

Recall that all the computations are at $\left(x_{0}, t_{0}\right)$ and $\left(x_{0}, t_{0}\right)$ is the maximum point, $t_{0} \leq T^{\prime}$, so we have

$$
(\eta F)\left(x, T^{\prime}\right) \leq(\eta F)\left(x_{0}, t_{0}\right) \leq 9+\frac{C_{4}}{R^{2}} T^{\prime}+\frac{18 \sqrt{C_{2}}}{R} \sqrt{T^{\prime}} .
$$

But $\eta \equiv 1$ on $B_{R}$, hence

$$
\sup _{x \in B_{R}}\left(\left|\nabla_{b} \varphi\right|^{2}+3 \varphi_{t}\right)\left(x, T^{\prime}\right) \leq \frac{C_{4}}{R^{2}}+\frac{18 \sqrt{C_{2}}}{R} \frac{1}{\sqrt{T^{\prime}}}+\frac{9}{T^{\prime}} .
$$

Now for any fixed time $t \in(0, \infty)$, by letting $R \rightarrow \infty$, one obtains

$$
\frac{\left|\nabla_{b} u\right|^{2}}{u^{2}}+3 \frac{u_{t}}{u} \leq \frac{9}{t}
$$

on $H^{1} \times[0, T)$.

(ii) If $\varphi_{t}\left(x_{0}, t_{0}\right) \geq 0$, then $t^{1 / 2}\left|\nabla_{b} \varphi\right| \leq F^{1 / 2}$. The above inequality leads to

$$
\eta F-\frac{18 \sqrt{C_{2}}}{R} t^{1 / 2}(\eta F)^{1 / 2}-\left(9+\frac{C_{4}}{R^{2}} t\right) \leq 0 .
$$

Hence

$$
\eta F \leq 9+\frac{C_{4}}{R^{2}} t+\frac{18 \sqrt{C_{2}}}{R} t^{1 / 2}(\eta F)^{1 / 2} .
$$

If $(\eta F) \leq 1$, then

$$
\eta F \leq 9+\frac{C_{4}}{R^{2}} t+\frac{18 \sqrt{C_{2}}}{R} t^{1 / 2} .
$$

Otherwise,

$$
\eta F \leq 9+\frac{C_{4}}{R^{2}} t+\frac{18 \sqrt{C_{2}}}{R} t^{1 / 2}(\eta F) .
$$

For fix $t$, we can choose $R$ such that $\frac{18 \sqrt{C_{2}}}{R} t^{1 / 2} \leq \frac{1}{2}$, thus

$$
\eta F \leq 18+\frac{C_{4}}{R^{2}} t
$$

and similar argument as before

$$
\sup _{x \in B_{R}}\left(\left|\nabla_{b} \varphi\right|^{2}+3 \varphi_{t}\right)\left(x, T^{\prime}\right) \leq \frac{C_{4}}{R^{2}}+\frac{18}{T^{\prime}} .
$$


Now for any fixed time $t \in(0, \infty)$, by letting $R \rightarrow \infty$ such that $\frac{18 \sqrt{C_{2}}}{R} t^{1 / 2} \rightarrow 0$, one obtains

$$
\frac{\left|\nabla_{b} u\right|^{2}}{u^{2}}+3 \frac{u_{t}}{u} \leq \frac{9+\epsilon}{t}
$$

on $H^{1} \times[0, T)$ for any $\epsilon>0$. $\square$

Then, by combining Theorem 4.2 and Proposition 4.1, Theorem 1.4 follows for $n=1$ easily.

Now we will apply the subgradient estimates in Theorem 4.2 and Proposition 4.1 to obtain the following Harnack inequality for positive solutions of the CR heat equation (1.6) on $H^{1} \times[0, T)$.

THEOREM 4.3. If $u(x, t)$ be a positive smooth solution of (1.6)

$$
\left(\Delta_{b}-\frac{\partial}{\partial t}\right) u(x, t)=0
$$

on $H^{1} \times[0, T)$ with

$$
P_{1} u=0
$$

at $t=0$, then for all points $x_{1}, x_{2}$ in $H^{1}$ and times $0<t_{1}<t_{2}<T$, we have the inequality

$$
\frac{t_{1}}{t_{2}} \exp \left(-\frac{d_{c}^{2}\left(x_{1}, x_{2}\right)}{4\left(t_{2}-t_{1}\right)}\right) \leq \frac{u\left(x_{2}, t_{2}\right)}{u\left(x_{1}, t_{1}\right)} \leq\left(\frac{t_{2}}{t_{1}}\right)^{(3+\epsilon)} \exp \left(\frac{3 d_{c}^{2}\left(x_{1}, x_{2}\right)}{4\left(t_{2}-t_{1}\right)}\right)
$$

for any $\epsilon>0$.

Proof. Let $\gamma$ be a horizontal curve with $\gamma\left(t_{1}\right)=x_{1}$ and $\gamma\left(t_{2}\right)=x_{2}$. We define $\eta:\left[t_{1}, t_{2}\right] \rightarrow M \times\left[t_{1}, t_{2}\right]$ by

$$
\eta(t)=(\gamma(t), t) .
$$

Clearly $\eta\left(t_{1}\right)=\left(x_{1}, t_{1}\right)$ and $\eta\left(t_{2}\right)=\left(x_{2}, t_{2}\right)$. Let $\varphi=\log u(x, t)$, integrate $\frac{d}{d t} \varphi$ along $\eta$, we get

$$
\begin{aligned}
\varphi\left(x_{2}, t_{2}\right)-\varphi\left(x_{1}, t_{1}\right) & =\int_{t_{1}}^{t_{2}} \frac{d}{d t} \varphi d t \\
& =\int_{t_{1}}^{t_{2}}\left\{\left\langle\dot{\gamma}, \nabla_{b} \varphi\right\rangle+\varphi_{t}\right\} d t .
\end{aligned}
$$

Applying Theorem 4.2 to $\varphi_{t}$, this yields

$$
\begin{aligned}
\varphi\left(x_{2}, t_{2}\right)-\varphi\left(x_{1}, t_{1}\right) & \leq \int_{t_{1}}^{t_{2}}\left\{|\gamma|\left|\nabla_{b} \varphi\right|+\varphi_{t}\right\} d t \\
& \leq \int_{t_{1}}^{t_{2}}\left\{\frac{3}{4} \mid \dot{\gamma}^{2}+\frac{3+\epsilon}{t}\right\} d t \\
& =\int_{t_{1}}^{t_{2}} \frac{3}{4}|\dot{\gamma}| d t+(3+\epsilon) \log \left(\frac{t_{2}}{t_{1}}\right) .
\end{aligned}
$$


Then the right-hand side inequality in theorem 4.3 follows by taking exponentials of the above inequality. Similarly, we can also get the left-hand side inequality.

As a consequence of Theorem 4.3 and $[\mathrm{CY}]$, we have

Corollary 4.4. Let $H(x, y, t)$ be a $L^{2}$-heat kernel of (1.6) on $H^{1} \times[0, T)$. Then for some constant $\delta$ and $0<\epsilon<1$, we have the inequality

$$
H(x, y, t) \leq \frac{C(\epsilon)^{\delta}}{V\left(B_{x}(\sqrt{t})\right)} \exp \left(-\frac{d_{c}^{2}(x, y)}{(4+\epsilon) t}\right) \leq \frac{C(\epsilon)^{\delta}}{t^{2}} \exp \left(-\frac{d_{c}^{2}(x, y)}{(4+\epsilon) t}\right)
$$

with $C(\epsilon) \rightarrow \infty$ as $\epsilon \rightarrow 0$.

REMARK 4.1. Here we use the volume $V\left(B_{x}(R)\right) \leq C R^{(2 n+2)}$ in an $(2 n+1)$ dimensional Heisenberg group $H^{n}([D T])$. One should compare this result with [BGG].

Then Corollary 1.6 follows easily from Theorem 1.4 and Corollary 4.4.

5. Sub-Laplacian of Carnot-Caratheodory distance on Heisenberg groups $H^{n}$. In this section, we prove the sub-Laplacian comparison $(*)$ as in previous section. We consider the following two vector fields defined on $\mathbf{R}^{3}$ with coordinates $(x, t)=\left(x_{1}, x_{2}, t\right)$ :

$$
X_{1}=\frac{\partial}{\partial x_{1}}+2 a x_{2} \frac{\partial}{\partial t} \quad \text { and } \quad X_{2}=\frac{\partial}{\partial x_{2}}-2 a x_{1} \frac{\partial}{\partial t}
$$

with $a>0$. It is easy to check that

$$
\left[X_{1}, X_{2}\right]=-4 a \frac{\partial}{\partial t} .
$$

Now we consider the following operator

$$
\Delta_{H}=-\frac{1}{2}\left(X_{1}^{2}+X_{2}^{2}\right)
$$

The vector fields $X_{1}, X_{2}$ and $T=\frac{\partial}{\partial t}$ and the operator $\Delta_{H}$ are left-invariant with respect to the "Heisenberg translation": for $(x, t)=\left(x_{1}, x_{2}, t\right)$ and $(y, s)=\left(y_{1}, y_{2}, s\right) \in$ $\mathbf{R}^{3}$,

$$
(x, t) \circ(y, s)=\left(x_{1}+y_{1}, x_{2}+y_{2}, t+s+2 a\left[x_{2} y_{1}-x_{1} y_{2}\right]\right) .
$$

Actually, the above multiplicative law defines a group structure on $\mathbf{R}^{3}$ which we call the 1-dimensional Heisenberg group with $(x, t)^{-1}=(-x,-t)$.

REMARK 5.1. By comparing the previous notations, we first put some conventions as followings: for $a=\frac{1}{2}$

$$
Z_{1}=\frac{1}{2}\left(X_{1}-i X_{2}\right) \text { and } Z_{\overline{1}}=\frac{1}{2}\left(X_{1}+i X_{2}\right)
$$

and

$$
J\left(X_{1}\right)=X_{2} \text { and } J\left(X_{2}\right)=-X_{1}
$$

and

$$
\Delta_{b}=-\Delta_{H} .
$$


The symbol of $\Delta_{H}$ is

$$
H(x, \xi, \theta)=\frac{1}{2}\left(\xi_{1}+2 a x_{2} \theta\right)^{2}+\frac{1}{2}\left(\xi_{2}-2 a x_{1} \theta\right)^{2}=\frac{1}{2}\left(\zeta_{1}^{2}+\zeta_{2}^{2}\right),
$$

where $\zeta_{1}=\xi_{1}+2 a x_{2} \theta$ and $\zeta_{2}=\xi_{2}-2 a x_{1} \theta$.

In this notation, Hamilton-Jacobi equations for the bicharacteristic curve $\left(x_{1}(s), x_{2}(s), t(s), \xi_{1}(s), \xi_{2}(s), \theta(s)\right)$ take the form:

$$
\begin{aligned}
\dot{x}_{1}(s) & =\frac{\partial H}{\partial \xi_{1}}=\xi_{1}+2 a x_{2} \theta=\zeta_{1}(s), \\
\dot{x}_{2}(s) & =\frac{\partial H}{\partial \xi_{2}}=\xi_{2}-2 a x_{1} \theta=\zeta_{2}(s), \\
\dot{t}(s) & =\frac{\partial H}{\partial \theta}=\left(\xi_{1}+2 a x_{2} \theta\right)\left(2 a x_{2}\right)-\left(\xi_{2}-2 a x_{1} \theta\right)\left(2 a x_{1}\right)=2 a\left(\zeta_{1} x_{1}-\zeta_{2} x_{2}\right), \\
\dot{\xi}_{1}(s) & =-\frac{\partial H}{\partial x_{1}}=(2 a \theta)\left(\xi_{2}-2 a x_{1} \theta\right)=(2 a \theta) \zeta_{2}, \\
\dot{\xi}_{2}(s) & =-\frac{\partial H}{\partial x_{2}}=-(2 a \theta)\left(\xi_{1}+2 a x_{2} \theta\right)=-(2 a \theta) \zeta_{1}, \\
\dot{\theta}(s) & =-\frac{\partial H}{\partial t}=0,
\end{aligned}
$$

where the dot denotes $\frac{d}{d s}$. We let $s$ run along the ray from 0 to a point $\tau \in \mathbf{C}$. Because of group invariance we need to consider paths relative to the origin and a point $(x, t)=\left(x_{1}, x_{2}, t\right)$ only, and assume boundary conditions

$$
x_{1}(0)=0, \quad x_{2}(0)=0, \quad x_{1}(\tau)=x_{1}, \quad x_{2}(\tau)=x_{2}, \quad t(\tau)=t .
$$

Then it is easy to see that the Hamiltonian,

$$
\frac{1}{2} \dot{x}_{1}^{2}(s)+\frac{1}{2} \dot{x}_{2}^{2}(s)=H(x, \xi, \theta)=H_{0} \equiv \frac{1}{2}\left(\zeta_{1}(0) \zeta_{1}(0)+\zeta_{2}(0) \zeta_{2}(0)\right) .
$$

is constant along a given bicharacteristic. The projection of the bicharacteristic curve onto the base is a subRiemannian geodesic connecting the point $(x, t)$ to the origin.

From (5.1), we know that $\theta(s)=\theta(0)=\theta$ and we may take it to be the free parameter. Equations (5.1) imply that

$$
\begin{aligned}
& \dot{\zeta}_{1}=\dot{\xi}_{1}+2 a \theta \dot{x}_{2}=2 a \theta \zeta_{2}+2 a \theta \zeta_{2}=4 a \theta \zeta_{2}, \\
& \dot{\zeta}_{2}=\dot{\xi}_{2}-2 a \theta \dot{x}_{1}=-2 a \theta \zeta_{1}-2 a \theta \zeta_{1}=-4 a \theta \zeta_{1} .
\end{aligned}
$$

Hence,

$$
\begin{aligned}
& \zeta_{1}(s)=\cos (4 a \theta s) \zeta_{1}(0)+\sin (4 a \theta s) \zeta_{2}(0), \\
& \zeta_{2}(s)=-\sin (4 a \theta s) \zeta_{1}(0)+\cos (4 a \theta s) \zeta_{2}(0) .
\end{aligned}
$$

Therefore, we may solve for $x(s)$ as a function of $x, \tau$ and $\theta$, and then solve for $t(s)$ as a function of $x, t, \tau$ and $\theta$. Here are the calculations.

$$
\begin{aligned}
x_{1}(s) & =\int_{0}^{s} \zeta_{1}(\rho) d \rho=-\frac{1}{4 a \theta}\left\{\zeta_{2}(s)-\zeta_{2}(0)\right\} \\
& =-\frac{1}{4 a \theta}\left\{-\sin (4 a \theta s) \zeta_{1}(0)+[\cos (4 a \theta s)-1] \zeta_{2}(0)\right\} \\
& =\frac{\sin (2 a \theta s)}{2 a \theta}\left\{\cos (2 a \theta s) \zeta_{1}(0)+\sin (2 a \theta s)\right\}
\end{aligned}
$$

and

$$
\begin{aligned}
x_{2}(s) & =\frac{1}{4 a \theta}\left\{\zeta_{1}(s)-\zeta_{1}(0)\right\} \\
& =\frac{1}{4 a \theta}\left\{[\cos (4 a \theta s)-1] \zeta_{1}(0)+\sin (4 a \theta s) \zeta_{2}(0)\right\} \\
& =\frac{\sin (2 a \theta s)}{2 a \theta}\left\{-\cos (2 a \theta s) \zeta_{1}(0)+\sin (2 a \theta s)\right\} .
\end{aligned}
$$


Therefore,

$$
\left[\begin{array}{l}
\zeta_{1}(0) \\
\zeta_{2}(0)
\end{array}\right]=\frac{2 a \theta}{\sin (2 a \theta \tau)}\left[\begin{array}{cc}
\cos (2 a \theta \tau) & -\sin (2 a \theta \tau) \\
\sin (2 a \theta \tau) & \cos (2 a \theta \tau)
\end{array}\right]\left[\begin{array}{l}
x_{1} \\
x_{2}
\end{array}\right]
$$

It follows that

$$
H_{0}=\frac{1}{2}\left(\zeta_{1}(0) \zeta_{1}(0)+\zeta_{2}(0) \zeta_{2}(0)\right)=\frac{(2 a \theta)^{2}}{2 \sin ^{2}(2 a \theta \tau)}\left(x_{1}^{2}+x_{2}^{2}\right)=\frac{(2 a \theta)^{2}}{2 \sin ^{2}(2 a \theta \tau)}\|x\|^{2} .
$$

When $\theta=0$, we have $\zeta(s)=\zeta(0), x(s)=\zeta(0) s$ and $t(s)=t(0)$. Substituting these calculations into (5.1), we have

$$
\begin{aligned}
t-t(s) & =2 a \int_{s}^{\tau}\left[\zeta_{1}(\rho) x_{2}(\rho)-\zeta_{2}(\rho) x_{1}(\rho)\right] d \rho \\
& =\frac{1}{2 \theta} \int_{s}^{\tau}[1-\cos (4 a \theta \rho)] d \rho \cdot\left[\zeta_{1}^{2}(0)+\zeta_{2}^{2}(0)\right] \\
& =(\tau-s) \frac{2 a^{2} \theta}{\sin ^{2}(2 a \theta \tau)}\|x\|^{2}-\frac{a}{2} \cdot \frac{\sin (4 a \theta \tau)-\sin (4 a \theta s)}{\sin ^{2}(2 a \theta \tau)}\|x\|^{2} .
\end{aligned}
$$

THEOREM 5.1. The solution of equations (5.1) with boundary conditions (5.2) is

$$
\begin{aligned}
x_{1}(s) & =\frac{\sin (2 a \theta s)}{\sin (2 a \theta \tau)}\left\{\cos [2 a \theta(s-\tau)] x_{1}+\sin [2 a \theta(s-\tau)] x_{2}\right\}, \\
& =\frac{\sin (2 a \theta s)}{\sin (2 a \theta \tau)}\left\{-\sin [2 a \theta(s-\tau)] x_{1}+\cos [2 a \theta(s-\tau)] x_{2}\right\}, \\
& =\left[\frac{a}{2} \frac{\sin (4 a \theta \tau)-\sin (4 a \theta s)}{\sin ^{2}(2 a \theta \tau)}-(\tau-s) \frac{2 a^{2} \theta}{\sin ^{2}(2 a \theta \tau)}\right]\left(x_{1}^{2}+x_{2}^{2}\right)-t .
\end{aligned}
$$

The value of the Hamiltonian $H$ on this path is

$$
H_{0}=\frac{2 a^{2} \theta^{2}}{\sin ^{2}(2 a \theta \tau)}\left(x_{1}^{2}+x_{2}^{2}\right) .
$$

Next (5.3) yields

$$
t-t(0)=a \mu(2 a \theta \tau)\|x\|^{2}
$$

where we set

$$
\mu(z)=\frac{z}{\sin ^{2} z}-\cot z .
$$

The action integral associated to the Hamiltonian curve is

$$
S(x, t, \tau ; \theta)=\int_{0}^{\tau}\left\{\sum_{j=1}^{2} \xi_{j}(s) \dot{x}_{j}(s)+\theta \dot{t}(s)-H(x(s), \xi(s), \theta)\right\} d s .
$$

$H$ is homogeneous of degree 2 with respect to $\left(\xi_{1}, \xi_{2}, \theta\right)$, so

$$
S=\int_{0}^{\tau}\left\{\sum_{j=1}^{2} \xi_{j} \frac{\partial H}{\partial \xi_{j}}+\theta \frac{\partial H}{\partial \theta}-H\right\} d s=\int_{0}^{\tau}(2 H-H) d s=\tau H_{0} .
$$

From formulas (5.3), we have the following theorem: 
THEOREM 5.2. The action integral $S(x, t, \tau, \theta)$ is given by

$$
\begin{aligned}
S(x, t, \tau, \theta) & =\frac{\tau(2 a \theta)^{2}}{2 \sin ^{2}(2 a \theta \tau)}\|x\|^{2}, \\
& =[t-t(0)] \theta+a \theta \cot (2 a \theta \tau)\left(x_{1}^{2}+x_{2}^{2}\right), \quad \theta \in\left[0, \frac{\pi}{a}\right) .
\end{aligned}
$$

It is convenient to fix $\tau, \tau=1$. Then the Hamiltonian paths are determined entirely by the parameter $\theta$. We may take the end points to be $(\mathbf{0}, 0)$ and $(x, t)$. Then $\theta$ must satisfy

$$
t=a \mu(2 a \theta)\left(x_{1}^{2}+x_{2}^{2}\right)=a \mu(2 a \theta)\|x\|^{2} .
$$

It can be shown that $\mu$ is a monotone increasing diffeomorphism of the interval $(-\pi, \pi)$ onto $\mathbf{R}$. On each interval $(m \pi,(m+1) \pi), m=1,2, \ldots, \mu$ has a unique critical point $z_{m}$. On this interval $\mu$ decreases strictly from $+\infty$ to $\mu\left(z_{m}\right)$ and then increases strictly from $\mu\left(z_{m}\right)$ to $+\infty$. Now the complete picture of the geodesics is given in the following two theorems.

THEOREM 5.3. There are finitely many geodesics that join the origin to $(x, t)$ if and only if $x \neq \mathbf{0}$. These geodesics are parametrized by the solutions $\theta$ of

$$
a \mu(2 a \theta)\|x\|^{2}=|t|
$$

and their lengths increase strictly with $\theta$. There is exactly one such geodesic if and only if

$$
|t|<a \mu\left(z_{1}\right)\|x\|^{2}
$$

and the number of geodesics increases without bound as $\frac{|t|}{a\|x\|^{2}} \rightarrow \infty$.

The square of the length of the geodesic associated to a solution $\theta$ of (5.5) is

$$
\begin{aligned}
2 S(x,|t|, 1, \theta) & =\frac{(2 a \theta)^{2}}{\sin ^{2}(2 a \theta)}\left(x_{1}^{2}+x_{2}^{2}\right) \\
& =\frac{(2 a \theta)^{2}}{\sin ^{2}(2 a \theta)} \frac{\left(x_{1}^{2}+x_{2}^{2}\right)}{\left(x_{1}^{2}+x_{2}^{2}\right)+|t| / a}\left[\frac{|t|}{a}+\left(x_{1}^{2}+x_{2}^{2}\right)\right] \\
& =\frac{(2 a \theta)^{2}}{\sin ^{2}(2 a \theta)} \frac{1}{1+\mu(2 a \theta)}\left[\frac{|t|}{a}+\left(x_{1}^{2}+x_{2}^{2}\right)\right] \\
& =\nu(2 a \theta)\left(\frac{|t|}{a}+\|x\|^{2}\right),
\end{aligned}
$$

where $\nu(0)=2$ and otherwise

$$
\nu(z)=\frac{z^{2}}{\sin ^{2} z} \frac{1}{1+\mu(z)}=\frac{z^{2}}{z+\sin ^{2} z-\sin z \cos z} .
$$

Consequently, if $2 a \theta \in(m \pi,(m+1) \pi)$ the length $d_{\theta}$ of the geodesic satisfies

$$
\frac{m^{2} \pi^{2}}{(m+1) \pi+2}\left(\frac{|t|}{a}+\|x\|^{2}\right)<\left(d_{\theta}\right)^{2}<\frac{(m+1)^{2} \pi^{2}}{m \pi}\left(\frac{|t|}{a}+\|x\|^{2}\right) .
$$

When $x=\mathbf{0}$, we need to find the Hamiltonian paths connecting the origin to $(0, t)$, i.e., $x_{1}(1)=0, x_{2}(1)=0, t(1)=t$. This implies that $\zeta_{1}(1)=\zeta_{1}(0)$ and $\zeta_{2}(1)=\zeta_{2}(0)$. It follows that

$$
\begin{aligned}
& \zeta_{1}(1)=\cos (4 a \theta) \zeta_{1}(0)+\sin (4 a \theta) \zeta_{2}(0)=\zeta_{1}(0), \\
& \zeta_{2}(1)=-\sin (4 a \theta) \zeta_{1}(0)+\cos (4 a \theta) \zeta_{2}(0)=\zeta_{2}(0) .
\end{aligned}
$$


This implies that

$$
\sin (4 a \theta)=0, \quad \text { and } \quad \cos (4 a \theta)=1
$$

i.e.,

$$
2 a \theta=m \pi, \quad \text { with } \quad m=1,2,3, \ldots
$$

In this case,

$$
t=\frac{1}{2 \theta}\left(\zeta_{1}^{2}(0)+\zeta_{2}^{2}(0)\right),
$$

therefore, $\theta \neq 0$ and $m \neq 0$ in (5.4). We also know that

$$
d_{m}^{2}=\frac{m \pi|t|}{a}
$$

Summarizing, we have the following theorem.

THEOREM 5.4. The geodesics that join the origin to a point $(0,0, t)$ have lengths $d_{1}, d_{2}, d_{3}, \ldots$, where

$$
d_{m}^{2}=\frac{m \pi|t|}{a}
$$

Since $x_{1}(1)=x_{2}(1)=0$, we may use $\left(\zeta_{1}(0), \zeta_{2}(0)\right)$ to obtain the geodesics as follows:

$$
\begin{aligned}
x_{1}^{(m)}(s) & =-\frac{1}{2 m \pi}\left\{-\sin (2 m \pi s) \zeta_{1}(0)+[\cos (2 m \pi s)-1] \zeta_{2}(0)\right\} \\
& =\left(\frac{t}{4 a m \pi}\right)^{\frac{1}{2}}\left\{\sin (2 m \pi s) \frac{\zeta_{1}(0)}{\|\zeta(0)\|}+[1-\cos (2 m \pi s)] \frac{\zeta_{2}(0)}{\|\zeta(0)\|}\right\},
\end{aligned}
$$

where $\|\zeta(0)\|=\sqrt{\zeta_{1}^{2}(0)+\zeta_{2}^{2}(0)}$. Similarly, we have

$$
\begin{aligned}
x_{2}^{(m)}(s) & =\frac{1}{2 m \pi}\left\{[\cos (2 m \pi s)-1] \zeta_{1}(0)+\sin (2 m \pi s) \zeta_{2}(0)\right\} \\
& =\left(\frac{t}{4 a m \pi}\right)^{\frac{1}{2}}\left\{[\cos (2 m \pi s)-1] \frac{\zeta_{1}(0)}{\|\zeta(0)\|}+\sin (2 m \pi s) \frac{\zeta_{2}(0)}{\|\zeta(0)\|}\right\},
\end{aligned}
$$

and

$$
t^{(m)}(s)=[2 m \pi s-\sin (2 m \pi s)] \frac{t}{2 m \pi} .
$$

This shows that for each fixed $m, m=1,2, \ldots$, the geodesics $\left(x_{1}^{(m)}(s), x_{2}^{(m)}(s), t^{(m)}(s)\right)$ can be parametrized by a unit vector $\zeta(0) /\|\zeta(0)\|$ on the unit circle. These curves lie in a cylinder around the $t$-axis whose radius is $\mathcal{O}(1 / \sqrt{m})$.

A special case of (5.6) is the square of the Carnot-Caratheodory distance $\left[d_{c}(x, t)\right]^{2}$ :

$$
\left[d_{c}(x, t)\right]^{2}=2 S\left(x,|t|, 1 ; \theta_{c}\right)=\left[\frac{2 a \theta_{c}}{\sin \left(2 a \theta_{c}\right)}\right]^{2}\|x\|^{2}=\nu\left(2 a \theta_{c}\right)\left(\frac{|t|}{a}+\|x\|^{2}\right)
$$


where $\theta_{c}$ is the unique solution of $a \mu(2 a \theta)\|x\|^{2}=|t|$ in the interval $[0, \pi / 2 a)$. Introduce a new parameter $\phi=2 a \theta_{c}$. Then the Carnot-Caratheodory distance between the origin and point $\left(x_{1}, x_{2}, t\right)$ can be expressed as

$$
d_{c}(x, t)=\frac{\phi}{\sin \phi}\|x\| \quad \text { with } \quad a \mu(\phi)\|x\|^{2}=|t| \quad \text { and } \quad \phi \in[0, \pi) .
$$

We will compute $\Delta_{H} d_{c}(x, t)$. In polar coordinates,

$$
-\Delta_{H}=\frac{1}{2}\left(\frac{\partial^{2}}{\partial r^{2}}+\frac{1}{r} \frac{\partial^{2}}{\partial r \partial \theta}+\frac{1}{r^{2}} \frac{\partial^{2}}{\partial \theta^{2}}\right)+2 a \frac{\partial^{2}}{\partial t \partial \theta}+2 a^{2} r^{2} \frac{\partial^{2}}{\partial t^{2}} .
$$

Since $d_{c}(x, t)$ depends only on $r=\|x\|=\sqrt{x_{1}^{2}+x_{2}^{2}}$, we have

$$
-\Delta_{H} d_{c}(x, t)=\left(\frac{1}{2} \frac{\partial^{2}}{\partial r^{2}}+2 a^{2} r^{2} \frac{\partial^{2}}{\partial t^{2}}\right) d_{c}(r, t) .
$$

Introduce a new variable $u=|t| / a r^{2}$, then

$$
d_{c}(r, t):=f_{c}(r, u)=\frac{\phi}{\sin \phi} r \quad \text { where } u \text { satisfies } \quad u=\mu(\phi)=\frac{\phi-\sin \phi \cos \phi}{\sin ^{2} \phi} .
$$

Hence

$$
-\Delta_{H} d_{c}(r, t)=\left(\frac{1}{2} \frac{\partial^{2}}{\partial r^{2}}+\frac{2}{r^{2}} \frac{\partial^{2}}{\partial u^{2}}\right) f_{c}(r, u)=\frac{2}{r} \frac{\partial^{2}}{\partial u^{2}}\left(\frac{\phi}{\sin \phi}\right),
$$

where $u$ is given by $u=\mu(\phi)$. Let $g(\phi)=\frac{\phi}{\sin \phi}$. Then

$$
\frac{d g}{d u}=\frac{d g}{d \phi} \cdot \frac{d \phi}{d u} \quad \text { and } \quad \frac{d^{2} g}{d u^{2}}=\frac{d^{2} g}{d \phi^{2}} \cdot\left(\frac{d \phi}{d u}\right)^{2}+\frac{d g}{d \phi} \cdot \frac{d^{2} \phi}{d u^{2}} .
$$

We next compute $\frac{d g}{d \phi}, \frac{d^{2} g}{d \phi^{2}}, \frac{d \phi}{d u}$ and $\frac{d^{2} \phi}{d u^{2}}$.

$$
\frac{d g}{d \phi}=\frac{\sin \phi-\phi \cos \phi}{\sin ^{2} \phi} \text { and } \quad \frac{d^{2} g}{d \phi^{2}}=\frac{\phi\left(1+\cos ^{2} \phi\right)-2 \sin \phi \cos \phi}{\sin ^{3} \phi} .
$$

Next $u=\mu(\phi)$ implies

$$
1=\mu^{\prime}(\phi) \frac{d \phi}{d u}, \quad \frac{d \phi}{d u}=\frac{1}{\mu^{\prime}(\phi)} \quad \text { and } \quad \frac{d^{2} \phi}{d u^{2}}=-\frac{\mu^{\prime \prime}(\phi)}{\left(\mu^{\prime 3}\right)} .
$$

We now compute $\mu^{\prime}(\phi)$ and $\mu^{\prime \prime}(\phi)$ from $\mu(\phi)=\phi \csc ^{2} \phi-\cot \phi$.

$$
\mu^{\prime 2} \phi-2 \phi \csc ^{2} \phi \cot \phi+\csc ^{2} \phi=2 \csc ^{2} \phi(1-\phi \cot \phi) .
$$

and

$$
\begin{aligned}
& \mu^{\prime \prime 2} \phi \cot \phi(1-\phi \cot \phi)+2 \csc ^{2} \phi\left(\phi \csc ^{2} \phi-\cot \phi\right) \\
& =2 \csc ^{2} \phi\left[\phi\left(3 \cot ^{2} \phi+1\right)-3 \cot \phi\right] .
\end{aligned}
$$

We finally compute $-\Delta_{h} f_{c}(r, u)=\frac{2}{r} \frac{d^{2}}{d u^{2}} g(\phi)$.

$$
\begin{aligned}
-\Delta_{H} f_{c}(r, u) & =\frac{2}{r}\left[\frac{d^{2} g}{d \phi^{2}} \cdot\left(\frac{d \phi}{d u}\right)^{2}+\frac{d g}{d \phi} \cdot \frac{d^{2} \phi}{d u^{2}}\right] \\
& =\frac{2}{r}\left[\frac{d^{2} g}{d \phi^{2}} \cdot \frac{1}{\left(\mu^{\prime 2}\right)}-\frac{d g}{d \phi} \frac{\mu^{\prime \prime}(\phi)}{\left(\mu^{\prime 3}\right)}\right] \\
& =\frac{2}{r\left(\mu^{\prime 2}\right)}\left[\frac{d^{2} g}{d \phi^{2}}-\frac{d g}{d \phi} \cdot \frac{\mu^{\prime \prime}(\phi)}{\mu^{\prime}(\phi)}\right] .
\end{aligned}
$$


We shall compute the term in $[\ldots]$ in term of $\phi$ first.

$$
\begin{aligned}
& \frac{d^{2} g}{d \phi^{2}}-\frac{d g}{d \phi} \cdot \frac{\mu^{\prime \prime}(\phi)}{\mu^{\prime}(\phi)} \\
= & \frac{\phi\left(1+\cos ^{2} \phi\right)-2 \sin \phi \cos \phi}{\sin ^{3} \phi}-\frac{\sin \phi-\phi \cos \phi}{\sin ^{2} \phi} \cdot \frac{2 \csc ^{2} \phi\left[\phi\left(3 \cot ^{2} \phi+1\right)-3 \cot \phi\right]}{2 \csc ^{2} \phi(1-\phi \cot \phi)} \\
= & \frac{\phi\left(1+\cos ^{2} \phi\right)-2 \sin \phi \cos \phi}{\sin ^{3} \phi}-\frac{\phi\left(3 \cot ^{2} \phi+1\right)-3 \cot \phi}{\sin \phi} \\
= & \frac{\phi\left(1+\cos ^{2} \phi\right)-2 \sin \phi \cos \phi-\phi\left(3 \cos ^{2} \phi+\sin ^{2} \phi\right)+3 \cos \phi \sin \phi}{\sin ^{3} \phi} \\
= & \frac{\sin \phi \cos \phi-\phi \cos ^{2} \phi}{\sin ^{3} \phi} .
\end{aligned}
$$

Hence we have

$$
\begin{aligned}
-\Delta_{H} f_{c}(r, u) & =\frac{2}{r\left(\mu^{2}\right.}\left[\frac{d^{2} g}{d \phi^{2}}-\frac{d g}{d \phi} \cdot \frac{\mu^{\prime \prime}(\phi)}{\mu^{\prime}(\phi)}\right] \\
& =\frac{\sin \phi \cos \phi-\phi \cos ^{2} \phi}{\sin ^{3} \phi} \cdot \frac{1}{2 r \csc ^{4} \phi(1-\phi \cot \phi)^{2}} \\
& =\frac{(1-\phi \cot \phi) \sin \phi \cos \phi}{2 r \csc \phi(1-\phi \cot \phi)^{2}} \\
& =\frac{\sin ^{2} \phi \cos \phi}{2 r(1-\phi \cot \phi)} .
\end{aligned}
$$

Since $d_{c}=\frac{\phi}{\sin \phi} r$

$$
-\Delta_{H} d_{c}=\frac{1}{2 d_{c}} \cdot \frac{\phi \sin ^{2} \phi \cos \phi}{\sin \phi-\phi \cos \phi}
$$

We next study the function $F(\phi)=\frac{\phi \sin ^{2} \phi \cos \phi}{2(\sin \phi-\phi \cos \phi)}$ where $\phi$ is given by

$$
a r^{2} \mu(\phi)=t \quad \text { with } \quad \mu(\phi)=\frac{\phi-\sin \phi \cos \phi}{\sin ^{2} \phi} .
$$

The function $F(\phi)$ is smooth on the interval $[0, \pi]$, decreasing from $\left[0, \phi_{m}\right]$ and increasing from $\left[\phi_{m}, \pi\right] . \phi_{m}$ is the unique critical point of $F(\phi)$ inside the interval $(0, \pi)$. $F(0)=3, F(\pi / 2)=F(\pi)=0$.

As $r \rightarrow 0$ with $t>0$ fixed, $\phi \rightarrow \pi^{-}$and the equation $a r^{2} \mu(\phi)=t$ implies

$$
\frac{a r^{2}}{t} \frac{\phi-\sin \phi \cos \phi}{\sin ^{2} \phi}=1 \text {. }
$$

This shows that

$$
\phi \rightarrow \pi \quad \text { and } \quad \sin \phi \sim\left(\frac{a \pi}{t}\right)^{1 / 2} r \quad \text { as } \quad r \rightarrow 0 .
$$

This implies (5.7) makes sense when $r=0$. This corresponds to $\phi=\pi$.

All these imply

$$
\Delta_{b} d_{c}=-\Delta_{H} d_{c} \leq \frac{3}{d_{c}}
$$


and then the Sub-Laplacian comparison $(*)$ follows.

We now turn to the study of the $(2 n+1)$-dimensional Heisenberg group $H^{n}$. The manifold is $\mathbf{R}^{2 n} \times \mathbf{R}$ and the group law is given by

$$
(\mathbf{x}, t) \circ(\mathbf{y}, s)=\left(\mathbf{x}+\mathbf{y}, t+s+2 \sum_{j=1}^{n} a_{j}\left[x_{2 j} y_{2 j-1}-x_{2 j-1} y_{2 j}\right]\right)
$$

for $a_{1}, a_{2}, \cdots, a_{n}$ are positive constants and numbered so that

$$
0<a_{1} \leq a_{2} \leq \cdots \leq a_{n}
$$

The vector fields

$$
\begin{aligned}
X_{2 j-1} & =\frac{\partial}{\partial x_{2 j-1}}+2 a_{j} x_{2 j} \frac{\partial}{\partial t} \\
X_{2 j} & =\frac{\partial}{\partial x_{2 j}}-2 a_{j} x_{2 j-1} \frac{\partial}{\partial t} \\
T & =\frac{\partial}{\partial t}
\end{aligned}
$$

are left-invariant and generate the Lie algebra. The associated Heisenberg subLaplacian is

$$
\Delta_{H}=-\frac{1}{2} \sum_{j=1}^{2 n} X_{j}^{2}
$$

The symbol of $\Delta_{H}$ is

$$
H(\mathbf{x}, \xi, \theta)=\frac{1}{2} \sum_{j=1}^{n}\left[\left(\xi_{2 j-1}+2 a_{j} x_{2 j} \theta\right)^{2}+\left(\xi_{2 j}-2 a_{j} x_{2 j-1} \theta\right)^{2}=\frac{1}{2}\left(\zeta_{1}^{2}+\zeta_{2}^{2}\right) .\right.
$$

We can find the bicharacteristic curve connecting the point $(\mathbf{x}, t)$ to the origin by solving the associated Hamilton's equations which take essentially the same form as (5.1). We will just list the formulae that we need and refer to [BGG] for details. The value of the Hamiltonian $H$ on the bicharacteristic curve is the constant:

$$
H_{0}=\sum_{j=1}^{n} \frac{2 a_{j}^{2} \theta^{2}}{\sin ^{2}\left(2 a_{j} \tau \theta\right)} r_{j}^{2}
$$

with $r_{j}^{2}=x_{2 j-1}^{2}+x_{2 j}^{2}$. The analogue of (5.5) is follows:

$$
t=\sum_{j=1}^{n} a_{j} \mu\left(2 a_{j} \tau \theta\right) r_{j}^{2}
$$

The action integral $S(\mathbf{x}, t, \tau ; \theta)$ takes a similar form:

$$
S(\mathbf{x}, t, \tau ; \theta)=\sum_{j=1}^{n} \frac{4 \tau a_{j}^{2} \theta^{2}}{\sin ^{2}\left(2 a_{j} \tau \theta\right)} r_{j}^{2}=t \theta+\sum_{j=1}^{n} a_{j} \theta \cot \left(2 a_{j} \tau \theta\right) r_{j}^{2} .
$$


When we study the classical action and Carnot-Caratheodory distance, we set $\tau=1$. In the case of $\mathbf{x} \neq 0$, there are finitely many geodesics from the origin to $(\mathbf{x}, t)$. The geodesics are indexed by the solutions of

$$
|t|=\sum_{j=1}^{n} a_{j} \mu\left(2 a_{j} \theta\right) r_{j}^{2}
$$

and their lengths increase with $\theta$. The Carnot-Caratheodory distance from the origin to $(\mathbf{x}, t)$ is

$$
d^{2}(\mathbf{x}, t)=2 S\left(\mathbf{x},|t|, 1 ; \theta_{c}\right)
$$

where $\theta_{c}$ is the unique solution of (5.8) in the interval $\left[0, \pi / 2 a_{n}\right)$.

In the isotropic case $a_{1}=a_{2}=\cdots=a_{n}$, the results of the previous computations for $n=1$ carry over with no change.

6. Subgradient estimate on higher dimensional pseudohermitian manifolds. Let $(M, J, \theta)$ be a closed pseudohermitian $(2 n+1)$-manifold for $n \geq 2$. In this section, we derive the $\mathrm{CR}$ version of parabolic Li-Yau gradient estimate for the positive solution $u(x, t)$ of $(1.6)$ on $M \times[0, \infty)$ for $n \geq 2$.

First, we derive the following inequalities which we need in the proof of Proposition 1.1 .

Lemma 6.1. Let $(M, J, \theta)$ be a closed pseudohermitian $(2 n+1)$-manifold. Let $f$ be a smooth real-valued function on $M$. Then

$$
\left|\left(\nabla^{H}\right)^{2} f\right|^{2} \geq 2 \sum_{\alpha, \beta=1}^{n}\left|f_{\alpha \beta}\right|^{2}+2 \sum_{\substack{\alpha, \beta=1 \\ \alpha \neq \beta}}^{n}\left|f_{\alpha \bar{\beta}}\right|^{2}+\frac{1}{2} \sum_{\alpha=1}^{n}\left|f_{\alpha \bar{\alpha}}+f_{\bar{\alpha} \alpha}\right|^{2} .
$$

Proof. Since

$$
\begin{aligned}
\left|\left(\nabla^{H}\right)^{2} f\right|^{2} & =2 \sum_{\alpha, \beta=1}^{n}\left(f_{\alpha \beta} f_{\bar{\alpha} \bar{\beta}}+f_{\alpha \bar{\beta}} f_{\bar{\alpha} \beta}\right) \\
& =2 \sum_{\alpha, \beta=1}^{n}\left(\left|f_{\alpha \beta}\right|^{2}+\left|f_{\alpha \bar{\beta}}\right|^{2}\right) \\
& =2\left(\sum_{\alpha, \beta=1}^{n}\left|f_{\alpha \beta}\right|^{2}+\sum_{\substack{\alpha, \beta=1 \\
\alpha \neq \beta}}^{n}\left|f_{\alpha \bar{\beta}}\right|^{2}+\sum_{\alpha=1}^{n}\left|f_{\alpha \bar{\alpha}}\right|^{2}\right)
\end{aligned}
$$

and

$$
\begin{aligned}
\sum_{\alpha=1}^{n}\left|f_{\alpha \bar{\alpha}}\right|^{2} & =\frac{1}{4} \sum_{\alpha=1}^{n}\left(\left|f_{\alpha \bar{\alpha}}+f_{\bar{\alpha} \alpha}\right|^{2}+f_{0}^{2}\right) \\
& =\frac{1}{4} \sum_{\alpha=1}^{n}\left|f_{\alpha \bar{\alpha}}+f_{\bar{\alpha} \alpha}\right|^{2}+\frac{n}{4} f_{0}^{2} .
\end{aligned}
$$

It follows that

$$
\begin{aligned}
\left|\left(\nabla^{H}\right)^{2} f\right|^{2} & =2\left(\sum_{\alpha, \beta=1}^{n}\left|f_{\alpha \beta}\right|^{2}+\sum_{\substack{\alpha, \beta=1 \\
\alpha \neq \beta}}^{n}\left|f_{\alpha \bar{\beta}}\right|^{2}\right)+\frac{1}{2} \sum_{\alpha=1}^{n}\left|f_{\alpha \bar{\alpha}}+f_{\bar{\alpha} \alpha}\right|^{2}+\frac{n}{2} f_{0}^{2} \\
& \geq 2\left(\sum_{\alpha, \beta=1}^{n}\left|f_{\alpha \beta}\right|^{2}+\sum_{\substack{\alpha, \beta=1 \\
\alpha \neq \beta}}^{n}\left|f_{\alpha \bar{\beta}}\right|^{2}\right)+\frac{1}{2} \sum_{\alpha=1}^{n}\left|f_{\alpha \bar{\alpha}}+f_{\bar{\alpha} \alpha}\right|^{2} .
\end{aligned}
$$

Lemma 6.2. Let $(M, J, \theta)$ be a closed pseudohermitian $(2 n+1)$-manifold for $n \geq 2$. Let $f$ be a smooth real-valued function on $M$. Then

$$
\begin{aligned}
\left\langle\nabla_{b} f, \nabla_{b}\left|\nabla_{b} f\right|^{2}\right\rangle \leq & (n+2) \sum_{\alpha, \beta=1}^{n}\left|f_{\alpha \beta}\right|^{2}+(n+2) \sum_{\substack{\alpha, \beta=1 \\
\alpha \neq \beta}}^{n}\left|f_{\alpha \bar{\beta}}\right|^{2} \\
& +\left(\Delta_{b} f+\left|\nabla_{b} f\right|^{2}\right)\left|\nabla_{b} f\right|^{2}+\frac{(n+2)(n-1)}{4(n+1)} \sum_{\alpha=1}^{n}\left|f_{\alpha \bar{\alpha}}+f_{\bar{\alpha} \alpha}\right|^{2} .
\end{aligned}
$$


Proof. We first derive

$$
\begin{aligned}
& \left\langle\nabla_{b} f, \nabla_{b}\left|\nabla_{b} f\right|^{2}\right\rangle \\
= & 4 \sum_{\alpha, \beta=1}^{n} \operatorname{Re}\left(f_{\alpha \beta} f_{\bar{\alpha}} f_{\bar{\beta}}+f_{\alpha \bar{\beta}} f_{\bar{\alpha}} f_{\beta}\right) \\
= & 4 \operatorname{Re}\left(\sum_{\alpha, \beta=1}^{n} f_{\alpha \beta} f_{\bar{\alpha}} f_{\bar{\beta}}+\sum_{\substack{\alpha, \beta=1 \\
\alpha \neq \beta}}^{n} f_{\alpha \bar{\beta}} f_{\bar{\alpha}} f_{\beta}\right)+2 \sum_{\alpha=1}^{n}\left(f_{\alpha \bar{\alpha}}+f_{\bar{\alpha} \alpha}\right)\left|f_{\alpha}\right|^{2} \\
\leq \quad & (n+2)\left(\sum_{\alpha, \beta=1}^{n}\left|f_{\alpha \beta}\right|^{2}+\sum_{\substack{\alpha, \beta=1 \\
\alpha \neq \beta}}^{n}\left|f_{\alpha \bar{\beta}}\right|^{2}\right)+\frac{4}{n+2} \sum_{\alpha, \beta=1}^{n}\left|f_{\alpha}\right|^{2}\left|f_{\beta}\right|^{2} \\
& +\frac{4}{n+2} \sum_{\substack{\alpha, \beta=1 \\
\alpha \neq \beta}}^{n}\left|f_{\alpha}\right|^{2}\left|f_{\beta}\right|^{2}+2 \sum_{\alpha=1}^{n}\left(f_{\alpha \bar{\alpha}}+f_{\bar{\alpha} \alpha}\right)\left|f_{\alpha}\right|^{2} \\
= & (n+2)\left(\sum_{\alpha, \beta=1}^{n}\left|f_{\alpha \beta}\right|^{2}+\sum_{\substack{\alpha, \beta=1 \\
\alpha \neq \beta}}^{n}\left|f_{\alpha \bar{\beta}}\right|^{2}\right)+\frac{1}{n+2}\left|\nabla_{b} f\right|^{4} \\
& +\frac{4}{n+2} \sum_{\substack{\alpha, \beta=1 \\
\alpha \neq \beta}}^{n}\left|f_{\alpha}\right|^{2}\left|f_{\beta}\right|^{2}+2 \sum_{\alpha=1}^{n}\left(f_{\alpha \bar{\alpha}}+f_{\bar{\alpha} \alpha}\right)\left|f_{\alpha}\right|^{2} .
\end{aligned}
$$

Here we used the identity $\sum_{\alpha, \beta=1}^{n}\left|f_{\alpha}\right|^{2}\left|f_{\beta}\right|^{2}=\left(\sum_{\alpha=1}^{n}\left|f_{\alpha}\right|^{2}\right)^{2}=\frac{1}{4}\left|\nabla_{b} f\right|^{4}$.

Now we compute the last term in the above inequality.

$$
\begin{aligned}
& \sum_{\alpha=1}^{n}\left(f_{\alpha \bar{\alpha}}+f_{\bar{\alpha} \alpha}\right)\left|f_{\alpha}\right|^{2} \\
= & {\left[\sum_{\alpha=1}^{n}\left(f_{\alpha \bar{\alpha}}+f_{\bar{\alpha} \alpha}\right)\right]\left(\sum_{\beta=1}^{n}\left|f_{\beta}\right|^{2}\right) } \\
& -\sum_{\alpha=1}^{n}\left(f_{\alpha \bar{\alpha}}+f_{\bar{\alpha} \alpha}\right)\left(\sum_{\substack{n=1 \\
\beta \neq \alpha}}^{n}\left|f_{\beta}\right|^{2}\right) \\
\leq & \frac{1}{2} \Delta_{b} f\left|\nabla_{b} f\right|^{2}+\sum_{\alpha=1}^{n}\left|f_{\alpha \bar{\alpha}}+f_{\bar{\alpha} \alpha}\right|\left(\sum_{\substack{\beta=1 \\
\beta \neq \alpha}}^{n}\left|f_{\beta}\right|^{2}\right) \\
\leq & \frac{1}{2} \Delta_{b} f\left|\nabla_{b} f\right|^{2}+\frac{(n-1)(n+2)}{8(n+1)} \sum_{\alpha=1}^{n}\left|f_{\alpha \bar{\alpha}}+f_{\bar{\alpha} \alpha}\right|^{2} \\
& +\frac{2(n+1)}{(n-1)(n+2)} \sum_{\alpha=1}^{n}\left(\sum_{\substack{\beta=1 \\
\beta \neq \alpha}}^{n}\left|f_{\beta}\right|^{2}\right)^{2} .
\end{aligned}
$$

Substituting the above inequality into (6.1), one obtains

$$
\begin{aligned}
& \left\langle\nabla_{b} f, \nabla_{b}\left|\nabla_{b} f\right|^{2}\right\rangle \\
\leq & (n+2)\left(\sum_{\alpha, \beta=1}^{n}\left|f_{\alpha \beta}\right|^{2}+\sum_{\substack{\alpha, \beta=1 \\
\alpha \neq \beta}}^{n}\left|f_{\alpha \bar{\beta}}\right|^{2}\right)+\Delta_{b} f\left|\nabla_{b} f\right|^{2} \\
& +\frac{(n-1)(n+2)}{4(n+1)} \sum_{\alpha=1}^{n}\left|f_{\alpha \bar{\alpha}}+f_{\bar{\alpha} \alpha}\right|^{2}+\frac{1}{n+2}\left|\nabla_{b} f\right|^{4} \\
& +\frac{4(n+1)}{(n+2)(n-1)} \sum_{\alpha=1}^{n}\left(\sum_{\substack{\beta=1 \\
\beta \neq \alpha}}^{n}\left|f_{\beta}\right|^{2}\right)^{2}+\frac{4}{n+2} \sum_{\substack{\alpha, \beta=1 \\
\alpha \neq \beta}}^{n}\left|f_{\alpha}\right|^{2}\left|f_{\beta}\right|^{2} \\
\leq & (n+2)\left(\sum_{\alpha, \beta=1}^{n}\left|f_{\alpha \beta}\right|^{2}+\sum_{\substack{\alpha, \beta=1 \\
\alpha \neq \beta}}^{n}\left|f_{\alpha \bar{\beta}}\right|^{2}\right)+\Delta_{b} f\left|\nabla_{b} f\right|^{2} \\
& +\frac{(n+2)(n-1)}{4(n+1)} \sum_{\alpha=1}^{n}\left|f_{\alpha \bar{\alpha}}+f_{\bar{\alpha} \alpha}\right|^{2}+\frac{1}{n+2}\left|\nabla_{b} f\right|^{4} \\
& +\frac{4(n+1)}{(n+2)(n-1)}\left(\sum_{\alpha=1}^{n}\left(\sum_{\substack{\beta=1 \\
\beta \neq \alpha}}^{n}\left|f_{\beta}\right|^{2}\right)^{2}+\sum_{\substack{\alpha, \beta=1 \\
\alpha \neq \beta}}^{n}\left|f_{\alpha}\right|^{2}\left|f_{\beta}\right|^{2}\right) \\
= & (n+2)\left(\sum_{\alpha, \beta=1}^{n}\left|f_{\alpha \beta}\right|^{2}+\sum_{\substack{\alpha, \beta=1 \\
\alpha \neq \beta}}^{n}\left|f_{\alpha \bar{\beta}}\right|^{2}\right)+\Delta_{b} f\left|\nabla_{b} f\right|^{2} \\
& +\frac{(n+2)(n-1)}{4(n+1)} \sum_{\alpha=1}^{n}\left|f_{\alpha \bar{\alpha}}+f_{\bar{\alpha} \alpha}\right|^{2}+\left|\nabla_{b} f\right|^{4} .
\end{aligned}
$$

Here we have used the identity

$$
\sum_{\alpha=1}^{n}\left(\sum_{\substack{\beta=1 \\ \beta \neq \alpha}}^{n}\left|f_{\beta}\right|^{2}\right)^{2}+\sum_{\substack{\alpha, \beta=1 \\ \alpha \neq \beta}}^{n}\left|f_{\alpha}\right|^{2}\left|f_{\beta}\right|^{2}=(n-1)\left(\sum_{\alpha=1}^{n}\left|f_{\alpha}\right|^{2}\right)^{2}=\frac{n-1}{4}\left|\nabla_{b} f\right|^{4} .
$$

This completes the proof of the Lemma. $\mathrm{Q}$

Now we can derive the following Proposition 1.1 which is exact form of Lemma 3.3 for $n \geq 2$. 
Proof of Proposition 1.1. First differentiating (1.7) w.r.t. the $t$-variable, we have

$$
\begin{aligned}
G_{t} & =\frac{1}{t} G+t\left[\left|\nabla_{b} \varphi\right|^{2}+\left(1+\frac{2}{n}\right) \varphi_{t}\right]_{t} \\
& =\frac{1}{t} G+t\left[2\left(1+\frac{1}{n}\right)\left|\nabla_{b} \varphi\right|^{2}+\left(1+\frac{2}{n}\right) \Delta_{b} \varphi\right]_{t} \\
& =\frac{1}{t} G+t\left[4\left(1+\frac{1}{n}\right)\left\langle\nabla_{b} \varphi, \nabla_{b} \varphi_{t}\right\rangle+\left(1+\frac{2}{n}\right) \Delta_{b} \varphi_{t}\right] .
\end{aligned}
$$

By using the CR version of Bochner formula (2.1) and Lemma 3.2, one obtains

$$
\begin{aligned}
\Delta_{b} G= & t\left(\Delta_{b}\left|\nabla_{b} \varphi\right|^{2}+\left(1+\frac{2}{n}\right) \Delta_{b} \varphi_{t}\right) \\
= & t\left[2\left|\left(\nabla^{H}\right)^{2} \varphi\right|^{2}+2\left(1+\frac{2}{n}\right)\left\langle\nabla_{b} \varphi, \nabla_{b} \Delta_{b} \varphi\right\rangle\right. \\
& +2[2 R i c-(n+2) \operatorname{Tor}]\left(\left(\nabla_{b} \varphi\right)_{\mathbf{C}},\left(\nabla_{b} \varphi\right)_{\mathbf{C}}\right) \\
& \left.-\frac{8}{n}\left\langle P \varphi+\bar{P} \varphi, d_{b} \varphi\right\rangle_{L_{\theta}^{*}}+\left(1+\frac{2}{n}\right) \Delta_{b} \varphi_{t}\right] \\
\geq & t\left[2\left|\left(\nabla^{H}\right)^{2} \varphi\right|^{2}+2\left(1+\frac{2}{n}\right)\left\langle\nabla_{b} \varphi, \nabla_{b} \Delta_{b} \varphi\right\rangle-l_{0}\left|\nabla_{b} \varphi\right|^{2}\right. \\
& \left.-\frac{8}{n}\left\langle P \varphi+\bar{P} \varphi, d_{b} \varphi\right\rangle_{L_{\theta}^{*}}+\left(1+\frac{2}{n}\right) \Delta_{b} \varphi_{t}\right] \\
= & t\left[2\left|\left(\nabla^{H}\right)^{2} \varphi\right|^{2}+2\left(1+\frac{2}{n}\right)\left\langle\nabla_{b} \varphi, \nabla_{b} \Delta_{b} \varphi\right\rangle-l_{0}\left|\nabla_{b} \varphi\right|^{2}\right. \\
& -\frac{8}{n} u^{-2}\left\langle P u+\bar{P} u, d_{b} u\right\rangle_{L_{\theta}^{*}}+\frac{4}{n} \varphi_{t}\left|\nabla_{b} \varphi\right|^{2} \\
& \left.+\frac{4}{n}\left\langle\nabla_{b} \varphi, \nabla_{b}\left|\nabla_{b} \varphi\right|^{2}\right\rangle+\left(1+\frac{2}{n}\right) \Delta_{b} \varphi_{t}\right] .
\end{aligned}
$$

Here we have used the inequalities

$$
[2 \operatorname{Ric}-(n+2) \operatorname{Tor}]\left(\left(\nabla_{b} \varphi\right)_{C},\left(\nabla_{b} \varphi\right)_{C}\right) \geq-l_{0}\left|\left(\nabla_{b} \varphi\right)_{C}\right|^{2}=-\frac{l_{0}}{2}\left|\nabla_{b} \varphi\right|^{2}
$$

and

$$
\varphi_{t}=\frac{u_{t}}{u}=\frac{\Delta_{b} u}{u}
$$

Applying the formula

$$
\Delta_{b} \varphi=\varphi_{t}-\left|\nabla_{b} \varphi\right|^{2}=\frac{n}{(n+2) t} G-\frac{2(n+1)}{n+2}\left|\nabla_{b} \varphi\right|^{2}
$$

and combining $(6.2),(6.3)$, we conclude

$$
\begin{aligned}
& \left(\Delta_{b}-\frac{\partial}{\partial t}\right) G \\
\geq & -\frac{1}{t} G+t\left[2\left|\left(\nabla^{H}\right)^{2} \varphi\right|^{2}+2\left(1+\frac{2}{n}\right)\left\langle\nabla_{b} \varphi, \nabla_{b} \Delta_{b} \varphi\right\rangle+\frac{4}{n}\left\langle\nabla_{b} \varphi, \nabla_{b}\left|\nabla_{b} \varphi\right|^{2}\right\rangle\right. \\
& \left.-4\left(1+\frac{1}{n}\right)\left\langle\nabla_{b} \varphi, \nabla_{b} \varphi_{t}\right\rangle-l_{0}\left|\nabla_{b} \varphi\right|^{2}+\frac{4}{n} \varphi_{t}\left|\nabla_{b} \varphi\right|^{2}-\frac{8}{n} u^{-2}\left\langle P u+\bar{P} u, d_{b} u\right\rangle_{L_{\theta}^{*}}\right] \\
= & -\frac{1}{t} G-\frac{2 n}{(n+2)}\left\langle\nabla_{b} \varphi, \nabla_{b} G\right\rangle+t\left[2\left|\left(\nabla^{H}\right)^{2} \varphi\right|^{2}-\frac{4}{n+2}\left\langle\nabla_{b} \varphi, \nabla_{b}\left|\nabla_{b} \varphi\right|^{2}\right\rangle\right. \\
& \left.-l_{0}\left|\nabla_{b} \varphi\right|^{2}+\frac{4}{n} \varphi_{t}\left|\nabla_{b} \varphi\right|^{2}-\frac{8}{n} u^{-2}\left\langle P u+\bar{P} u, d_{b} u\right\rangle_{L_{\theta}^{*}}\right] .
\end{aligned}
$$


Now, by Lemma 6.1, Lemma 6.2, Cauchy-Schwarz inequality and applying the formula (6.4), we final have

$$
\begin{aligned}
& \left(\Delta_{b}-\frac{\partial}{\partial t}\right) G \\
\geq & -\frac{2 n}{(n+2)}\left\langle\nabla_{b} \varphi, \nabla_{b} G\right\rangle+t\left[\frac{2}{n+1} \sum_{\alpha=1}^{n}\left|\varphi_{\alpha \bar{\alpha}}+\varphi \bar{\alpha} \alpha\right|^{2}+\frac{8}{n(n+2)} \varphi_{t}\left|\nabla_{b} \varphi\right|^{2}\right. \\
& \left.-l_{0}\left|\nabla_{b} \varphi\right|^{2}-\frac{8}{n} u^{-2}\left\langle P u+\bar{P} u, d_{b} u\right\rangle_{L_{\theta}^{*}}\right]-\frac{1}{t} G \\
\geq & -\frac{2 n}{(n+2)}\left\langle\nabla_{b} \varphi, \nabla_{b} G\right\rangle+t\left[\frac{2}{n(n+1)}\left(\Delta_{b} \varphi\right)^{2}+\frac{8}{n(n+2)} \varphi_{t}\left|\nabla_{b} \varphi\right|^{2}\right. \\
& \left.-l_{0}\left|\nabla_{b} \varphi\right|^{2}-\frac{8}{n} u^{-2}\left\langle P u+\bar{P} u, d_{b} u\right\rangle_{L_{\theta}^{*}}\right]-\frac{1}{t} G \\
= & -\frac{2 n}{(n+2)}\left\langle\nabla_{b} \varphi, \nabla_{b} G\right\rangle+t\left[\frac{2 n}{(n+1)(n+2)^{2} t^{2}} G^{2}+\frac{8}{n(n+2)^{2}}\left|\nabla_{b} \varphi\right|^{4}\right. \\
& \left.-l_{0}\left|\nabla_{b} \varphi\right|^{2}-\frac{8}{n} u^{-2}\left\langle P u+\bar{P} u, d_{b} u\right\rangle_{L_{\theta}^{*}}\right]-\frac{1}{t} G .
\end{aligned}
$$

This completes the proof of Proposition 1.1.

Following the same proof as in Theorem 3.4. We have the following result.

Theorem 6.3. Let $(M, J, \theta)$ be a closed pseudohermitian $(2 n+1)$-manifold of zero torsion and nonnegative pseudohermitian Ricci tensors for $n \geq 2$. If $u(x, t)$ is the positive solution of (1.6) on $M \times[0, \infty)$ such that

$$
P_{\beta} u=0
$$

at $t=0$. Then $u$ satisfies the estimate

$$
\frac{\left|\nabla_{b} u\right|^{2}}{u^{2}}+\frac{n+2}{n} \frac{u_{t}}{u} \leq \frac{(n+1)(n+2)^{2}}{2 n} \frac{1}{t}
$$

on $M \times[0, \infty)$.

Following the same proof as in Theorem 4.2. We have the following result.

THEOREM 6.4. If $u(x, t)$ be a positive smooth solution of (1.6)

$$
\left(\Delta_{b}-\frac{\partial}{\partial t}\right) u(x, t)=0
$$

on $H^{n} \times[0, T)$ with

$$
P_{\beta} u=0
$$

at $t=0$, then $u$ satisfies the subgradient estimate

$$
\frac{\left|\nabla_{b} u\right|^{2}}{u^{2}}+\frac{n+2}{n} \frac{u_{t}}{u} \leq\left[\frac{(n+1)(n+2)^{2}}{2 n}+\epsilon\right] \frac{1}{t}
$$

on $H^{n} \times[0, T)$ for any $\epsilon>0$.

Then by combining Theorem 6.4 and Proposition 4.1, Theorem 1.4 follows easily for all $n$. 
[BCT] C. Berenstein, D. C. Chang And J. Tie, Laguerre Calculus and Its Applications in the Heisenberg Group, AMS/IP series in advanced mathematics \#22, International Press, Cambridge, Massachusetts, 2001.

[BGG] R. Beals, B. Gaveau and P. C. Greiner, Hamiltonian-Jacobi theory and the heat kernel on Heisenberg groups, J. Math. Pures Appl., 79 (2000), pp. 633-689.

[CC] S.-C. Chang And H.-L. Chiu, On the estimate of first eigenvalue of a sublaplacian on a pseudohermitian 3-manifold, Pacific J. Math., 232:2 (2007), pp. 269-282.

[CC1] S.-C. Chang And H.-L. ChiU, Nonnegativity of CR Paneitz operator and its Application to the CR Obata's Theorem in a Pseudohermitian $(2 n+1)$-Manifold, Journal of Geometric Analysis, 19 (2009), pp. 261-287.

[CC2] S.-C. Chang And H.-L. Chiu, On the CR Analogue of Obata's Theorem in a Pseudohermitian 3-Manifold, Math. Ann., 345:1 (2009), pp. 33-51.

[CCC] S.-C., Chang, J.-H. Cheng And H.-L. Chiu, The Fourth-order Q-curvature flow on a CR 3-manifold, Indiana Univ. Math. J., 56:4 (2007), pp. 1793-1826.

[CCW] S.-C. Chang, H.-L. Chiu And C.-T. Wu, The Li-Yau-Hamilton inequality for Yamabe flow on a closed CR 3-manifold, Trans. AMS., 362 (2010), pp. 1681-1698.

[CCh] S.-C., Chang And J.-H. Cheng, The Harnack Estimate for the Yamabe Flow on CR Manifolds of Dimension 3, Annals of Global Analysis and Geometry, 21:2 (2002), pp. 111121.

[Chi] H.-L. CHIU, The sharp lower bound for the first positive eigenvalue of the sublaplacian on a Pseudohermitian 3-manifold, Ann. Glob. Anal. Geom., 30 (2006), pp. 81-96.

[Cho] W.-L. CHOw, Uber System Von Lineaaren Partiellen Differentialgleichungen erster Orduung, Math. Ann., 117 (1939), pp. 98-105.

[CY] H.-D. CAO AND S.-T. YAU, Gradient Estimates, Harnack Inequalities and Estimates for Heat Kernels of the Sum of Squares of Vector Fields, Math. Z., 211 (1992), pp. 485504.

[DT] S. Dragomir and G. Tomassini, Differential Geometry and Analysis on CR Manifolds, Kirkhauser, Boston-Basel-Berlin, 2006.

[FH] C. Fefferman and K. Hirachi, Ambient Metric Construction of Q-Curvature in Conformal and CR Geometries, Math. Res. Lett., 10:5-6 (2003), pp. 819-831.

[GG] A. R. Gover And C. R. Graham, CR Invariant Powers of the Sub-Laplacian, J. Reine Angew. Math., 583 (2005), pp. 1-27.

[GL] C. R. Graham and J. M. LeE, Smooth Solutions of Degenerate Laplacians on Strictly Pseudoconvex Domains, Duke Math. J., 57 (1988), pp. 697-720.

[Gr] A. Greenleaf, The first eigenvalue of a Sublaplacian on a Pseudohermitian manifold, Comm. Part. Diff. Equ., 10(2):3 (1985), pp. 191-217.

[H] K. HiRAChI, Scalar Pseudo-hermitian Invariants and the Szegö Kernel on 3-dimensional CR Manifolds, Lecture Notes in Pure and Appl. Math. 143, pp. 67-76, Dekker, 1992.

[Ha] R. S. Hamilton, A Matrix Harnack Estimate for the Heat Equation, CAG, 1 (1993), pp. 113-126.

[KS] A. Koranyi and N. Stanton, Liouville Type Theorems for Some Complex Hypoelliptic Operators, J. Funct. Anal., 60 (1985), pp. 370-377.

[JSC] D. Jerison and A. SÁnchez-Calle, Estimates for the Heat Kernel for A Sum of Squares of Vector Fields, IUMJ, 35:4 (1986), pp. 835-854.

[LY] P. Li And S.-T. YAu, On the Parabolic Kernel of the Schrödinger Operator, Acta Math., 156 (1985), pp. 153-201.

[L1] J. M. LeE, Pseudo-Einstein Structure on CR Manifolds, Amer. J. Math., 110 (1988), pp. $157-178$.

[P] S. Paneitz, A Quartic Conformally Covariant Differential Operator for Arbitrary PseudoRiemannian Manifolds, preprint, 1983.

[PP] A. PASCUCCI AND S. Polidoro, On the Harnack Inequality for a Class of Hypoelliptic Evolution Equations, Trans. AMS, 356 (2004), pp. 4383-4394.

[SC] A. SÁnchez-Calle, Fundamental Solutions and Geometry of the Sum of Squares of Vector Fields, Invent. Math., 78 (1984), pp. 143-160.

[SY] R. Schoen And S.-T. YAu, Lectures on Differential Geometry, International Press, 1994.

[T] N. Tanaka, A differential Geometric Study on Strongly Pseudo-Convex Manifolds, Kinokuniya Book Store Co., Ltd, Kyoto, 1975.

[W] J.-P. WANG, Lecture Note in Geometric Analysis, NCTS, Hsinchu, Taiwan, 2005.

[We] S. M. Webster, Pseudohermitian Structures on a Real Hypersurface, J. Diff. Geom., 13 (1978), pp. 25-41. 
[Y] S. -T. YAU, Harmonic functions on complete Riemannian manifolds, Comm. Pure Appl. Math., 28 (1975), pp. 201-228. 